\title{
Analysis of Expression and Bioinformatics of Trehalose-6-phosphate Phosphatases (TPP) Gene Family in Wheat Indicates a Role in Plant Development and Stress Response
}

\author{
Linying Du \\ Northwest A\&F University \\ Shumin Li \\ Northwest A\&F University \\ Li Ding \\ Northwest A\&F University \\ Xinxiu Cheng \\ Northwest A\&F University \\ Zhensheng Kang \\ Northwest A\&F University \\ Hude Mao ( $\square$ mao_dehu@nwsuaf.edu.cn ) \\ Northwest A\&F University
}

\section{Research Article}

Keywords: TaTPPs, phylogenic relationship, expression profiles, plant development, stress response, TaTPP11

Posted Date: July 27th, 2021

DOI: https://doi.org/10.21203/rs.3.rs-663694/v1

License: (c) (i) This work is licensed under a Creative Commons Attribution 4.0 International License. Read Full License 


\section{Abstract}

Background: Trehalose-6-phosphate phosphatases genes (TPPS) are involved in the development and stress response of plants by regulating the biosynthesis of trehalose, though little is currently known about TPPs in common wheat (Triticum aestivum L.).

Results: In this study, we performed a genome-wide identification of the TPP gene family in common wheat, and identified a total of 31 TPP genes. These were subdivided into six subfamilies based on the phylogenetic relationships and the conservation of protein in six monocot and eudicot plants. The majority of TPP genes were represented by 2-3 wheat homoalleles (named TaTPPX ZA, TaTPPX_ZB, or TaTPPX_ZD), where Z is the location on the wheat chromosome of the gene number $(X)$. We also analyzed the chromosomal location, exon-intron structure, orthologous genes, and protein motifs of the TaTPPS. The RNA-seq data was used to perform an expression analysis, which found 26 TaTPP genes to be differentially expressed based on spatial and temporal characteristics, indicating they have varied functions in the growth and development of wheat.

Additionally, we assessed how the promoter regulatory elements were organized and used qRT-PCR in the leaves to observe how they were expressed following ABA, salt, low tempreture, and drought stress treatments. All of these genes exhibited differential expression against one or more stress treatments. Furthermore, overexpressing TaTPP11 in Arabidopsis results delayed plant development and enhanced drought tolerance, but not affect seed morphology.

Conclusions: TaTPPs could serve important roles in the development and stress response in wheat. These results provide a basis for subsequent research into the function of TaTPPS.

\section{Background}

Improving crop yields under both positive and negative field conditions is needed to increase worldwide food security. However, increasing the resilience and potential of crop yields at the same time is difficult, since the factors that responsible for stress tolerance and productivity are typically at odds with each other [1]. Carbon allocation and use can improve crop yields under variable conditions. Trehalose, a non-reducing disaccharide, is found in algae, invertebrates, bacteria, plants, fungi, and invertebrates [2]. The fact that trehalose is present in such a variety of life forms, coupled with the various biosynthetic pathways in each, indicates that trehalose metabolism serves an important evolutionary role by guarding the structure of cells and bioactive materials (including nucleic acids, membranes, and proteins) under environmental stressors (including freezing, oxidative, low-temperature, high-temperature, high-saline, and drought conditions) [3-5].

Plants typically only accumulate small levels of trehalose and its intermediates, making it unlikely to play an osmoprotective role [4]. Instead, the trehalose metabolic pathway and its related intermediates detect and regulate energetic status [2, 6]. For example, when it is exogenously applied, trehalose changes the physiology of the organism as well as its gene expression (including the induction of $A G P a s e$ genes found in Arabidopsis [7]), and increases the drought tolerance and biomass yield [8-10]. The ramosa3 mutant of Zea mays have significantly reduced trehalose and results excessive branching $[11,12]$. Trehalose-6-phosphate (T6P) is an intermediate of the trehalose metabolic pathway and serves an important roles during the signaling of plant sugars, assists in the regulation of the use and allotment of sucrose, and regulates the growth and development of crops $[2,6,13-15]$. Inducing an increase in T6P decreases the degradation of starch in Arabidopsis, while T6P alterations regulate flowering patterns and the photoperiod [16]. Recent studies have demonstrated that the relationship between SnRK1 (SNF1-related/AMPK protein kinases) and T6P can significantly alter how carbon is used and allotted in plants. Raising the levels of T6P can induce flux via the biosynthetic pathways responsible for yield and growth, while lowering the levels of T6P can mobilize carbon stores and induce the transport of carbon related to stress response $[1,2,14,17]$. As such, T6P can be targeted at particular types of tissues and cells during specific developmental stages to increase the resilience and potential of crop yields.

Plants synthesize trehalose by way of a conserved, two-step metabolic pathway. The first step entails the catalyzation of glucose from UDP-glucose to glucose 6-phosphate (G6P) via trehalose-6-phosphate synthase (TPS), resulting in trehalose-6-phosphate (T6P). The T6P is then dephosphorylated into trehalose via trehalose-6-phosphate phosphatase (TPP) [18]. The TPP and TPS genes have been found in species from all major plant taxa, suggesting that the metabolism of trehalose is likely found throughout the plant kingdom [7, 19-21]. Eleven TPS genes are encoded by the rice and Arabidopsis genomes, while 10 and 13 TPP genes are encoded by the rice and Arabidopsis genomes, respectively. There are two kinds of TPS genes: Class I TPS genes are typically single-copies and encode catalytically active TPS enzymes with both TPP and TPS domains possessing inactive phosphatase boxes; Class II TPS genes possess TPP and TPS domains but lack the TPS domain residue necessary to relate to the substrate. The majority of Class II TPS genes possess conserved phosphatase domains, but do not have any TPP or TPS activity [23]. TPP genes in all plants are comprised of a particular TPP domain that has conserved phosphatase domains, while all encode functional TPP enzymes in Arabidopsis. TPP genes share similar activities but differ in their patterns of differential expression, suggesting they could have a function related to specific tissues, stages, or processes [24].

A few TPP genes have recently been associated with abiotic stress responses. AtTPPD is a plastidial isoform regulated by redox reactions and associated with oxidative stress and salt resistance in Arabidopsis [22], while AtTPPF and AtTPP/ are two isoforms associates with drought response [25, 26]. OsTPP1 and OSTPP2 are induced by cold stress in rice [27, 28], while the OsTPP7 gene helps resist anaerobiosis during the germination stage in rice. This trait has been lost from many kinds of commercial varieties, along with the OsTPP7gene [29]. The MADS6 promoter

Page $2 / 24$ 
is active during the flowering of reproductive tissue and contributes to the expression of the OsTPP1 gene. This allows for significant improvements in both grain set and yield during the flowering stage under various drought conditions [13]. A number of TPP proteins serve important roles during plant development. For example, losing maize RAMOSA3 and ZmTPP4 reduces the determination of the meristem and increases inflorescence branching $[11,30]$.

Bread wheat (Triticum aestivum; $2 \mathrm{n}=6 \mathrm{x}=42$; $\mathrm{AABBDD}$ ) is widely grown and eaten around the globe [31]. The TPP genes play important roles in the development and stress response in plants. As such, we conducted a genome-wide analysis of the identification and expression of TPP genes in wheat. We produced a phylogenetic tree to assess the evolutionary relationships of TPP with wheat and other species of plants. We analyzed the conserved motifs, gene structures, and expression patterns in the stems, leaves, flag leaves, roots, spikes and grains across various developmental stages, as well as the cis-regulatory elements of the promoter sequences and ABA, salt, low tempreture, and drought stress responses in wheat. Additionally, we found that TaTPP11 transgenic overexpressing Arabidopsis slowed plant development and increased transgenic drought tolerance. This study provides a basis for subsequent research on the function of TaTPP genes.

\section{Results}

\section{Identification of the TPP gene family in wheat}

We obtained the wheat genome data used by this study from the Chinese Spring IWGSC RefSeq v1.1 reference genome assembly (https://wheaturgi.versailles.inra.fr/). First, we used a UNIX pipeline to convert the wheat genome to a local BLAST database. We then used 23 TPP protein sequences from Arabidopsis and rice to execute a BLAST search (BLASTP) with the local blast database, using a cut-off $E$-value $<1 \mathrm{e}^{-10}$. After filtering redundant sequences, we analyzed the remaining protein sequences and used the Simple Modular Architecture Research Tool (SMART; http://smart.embl-heidelberg.de/smart/set_mode.cgi?NORMAL=1). to identify the TPP domain. Lastly, we identified 31 TPP domain containing proteins in the most recent wheat genome (Additional file 1: Table S1). Of these, we assigned 11 clusters to various $A, B$, or $D$ sub-genomes, which we considered to be homoeologous copies of a single TPP gene. Wheat TPP genes were labeled as TaTPPX_ZA, TaTPPX_ZB, or TaTPPX_ZD, and Z denote the location on the wheat chromosome where the gene number $(X)$ is located. The detailed information of TaTPP genes in wheat was listed in Table 1. As shown in Table 1, the identified TaTPP genes in wheat encode proteins ranging from 249 (TaTPP5-2A) to 584 (TaTPP7-3D) amino acids (aa) in length with an average of 386 aa. Furthermore, the computed molecular weights of these TaTPP proteins ranged from 28.66 (TaTPP52A) to 96.02 (TaTPP7-3D) kDa. The theoretical pl of the deduced TaTPP proteins ranged from 5.53 (TaTPP1-1B and TaTPP1-1D) to 9.26 (TaTPP10$6 A)$. 
Table 1

Information on wheat TaTPP genes

\begin{tabular}{|c|c|c|c|c|c|c|c|c|c|c|c|}
\hline \multirow{2}{*}{$\begin{array}{l}\text { Gene } \\
\text { name }\end{array}$} & \multirow[t]{2}{*}{ Locus_ID } & \multirow[t]{2}{*}{ Chr. } & \multicolumn{2}{|l|}{ Position (bp) } & \multirow[t]{2}{*}{ ORF } & \multirow[t]{2}{*}{ Introns } & \multicolumn{3}{|c|}{ Deduced polypeptide } & \multicolumn{2}{|c|}{ Trehalose_Ppase } \\
\hline & & & Start & End & & & $\begin{array}{l}\text { Length } \\
\text { (aa) }\end{array}$ & MW (Da) & pl & $\begin{array}{l}\text { Start } \\
\text { (aa) }\end{array}$ & $\begin{array}{l}\text { End } \\
\text { (aa) }\end{array}$ \\
\hline $\begin{array}{l}\text { TaTPP1- } \\
1 \mathrm{~A}\end{array}$ & TraesCS1A02G210400 & $1 \mathrm{~A}$ & 372639121 & 372643307 & 1146 & 9 & 381 & 42614.46 & 5.61 & 119 & 364 \\
\hline $\begin{array}{l}\text { TaTPP1- } \\
\text { 1B }\end{array}$ & TraesCS1B02G224300 & 1B & 402147526 & 402150391 & 1146 & 12 & 381 & 42660.51 & 5.53 & 119 & 364 \\
\hline $\begin{array}{l}\text { TaTPP1- } \\
\text { 1D }\end{array}$ & TraesCS1D02G213700 & 1D & 298692275 & 298696295 & 1146 & 9 & 381 & 42625.49 & 5.53 & 119 & 364 \\
\hline $\begin{array}{l}\text { TaTPP2- } \\
2 \mathrm{~A}\end{array}$ & TraesCS2A02G161000 & $2 \mathrm{~A}$ & 111921839 & 111925164 & 1074 & 8 & 357 & 39430.21 & 6.11 & 106 & 339 \\
\hline $\begin{array}{l}\text { TaTPP2- } \\
2 \mathrm{~B}\end{array}$ & TraesCS2B02G187000 & $2 \mathrm{~B}$ & 161722263 & 161725704 & 1077 & 8 & 358 & 39621.52 & 6.04 & 107 & 340 \\
\hline $\begin{array}{l}\text { TaTPP2- } \\
\text { 2D }\end{array}$ & TraesCS2D02G168100 & $2 \mathrm{D}$ & 111588533 & 111591881 & 1077 & 8 & 358 & 39664.41 & 5.77 & 107 & 340 \\
\hline $\begin{array}{l}\text { TaTPP3- } \\
2 \mathrm{~A}\end{array}$ & TraesCS2A02G161100 & $2 \mathrm{~A}$ & 112744717 & 112747766 & 1077 & 8 & 358 & 39625.56 & 7.14 & 107 & 340 \\
\hline $\begin{array}{l}\text { TaTPP3- } \\
\text { 2B }\end{array}$ & TraesCS2B02G187100 & $2 \mathrm{~B}$ & 162007594 & 162010969 & 1077 & 8 & 358 & 39606.47 & 6.57 & 107 & 340 \\
\hline $\begin{array}{l}\text { TaTPP3- } \\
\text { 2D }\end{array}$ & TraesCS2D02G168200 & $2 \mathrm{D}$ & 112099169 & 112102442 & 1077 & 8 & 358 & 39503.35 & 6.84 & 107 & 340 \\
\hline $\begin{array}{l}\text { TaTPP4- } \\
2 \mathrm{~A}\end{array}$ & TraesCS2A02G161200 & $2 \mathrm{~A}$ & 113309228 & 113312068 & 1077 & 8 & 358 & 39632.62 & 6.77 & 107 & 340 \\
\hline $\begin{array}{l}\text { TaTPP4- } \\
2 \mathrm{~B}\end{array}$ & TraesCS2B02G187200 & $2 \mathrm{~B}$ & 162445597 & 162448929 & 1077 & 8 & 358 & 39653.69 & 7.12 & 107 & 340 \\
\hline $\begin{array}{l}\text { TaTPP4- } \\
\text { 2D }\end{array}$ & TraesCS2D02G168300 & $2 \mathrm{D}$ & 112177996 & 112181524 & 1179 & 9 & 392 & 43583.32 & 6.44 & 141 & 374 \\
\hline $\begin{array}{l}\text { TaTPP5- } \\
2 \mathrm{~A}\end{array}$ & TraesCS2A02G167100 & $2 \mathrm{~A}$ & 119307539 & 119314162 & 750 & 8 & 249 & 28665.56 & 8.28 & 2 & 230 \\
\hline $\begin{array}{l}\text { TaTPP5- } \\
\text { 2B }\end{array}$ & TraesCS2B02G193300 & $2 \mathrm{~B}$ & 168831609 & 168853380 & 1680 & 11 & 559 & 62281.71 & 8.61 & 312 & 540 \\
\hline $\begin{array}{l}\text { TaTPP6- } \\
2 \mathrm{~A}\end{array}$ & TraesCS2A02G412100 & $2 \mathrm{~A}$ & 669749666 & 669753186 & 1113 & 9 & 370 & 41228.87 & 5.70 & 112 & 347 \\
\hline $\begin{array}{l}\text { TaTPP6- } \\
2 \mathrm{~B}\end{array}$ & TraesCS2B02G430700 & $2 \mathrm{~B}$ & 619679522 & 619682850 & 1143 & 9 & 380 & 42099.78 & 5.75 & 112 & 348 \\
\hline $\begin{array}{l}\text { TaTPP6- } \\
2 \mathrm{D}\end{array}$ & TraesCS2D02G409300 & $2 \mathrm{D}$ & 524105415 & 524108680 & 1113 & 7 & 370 & 41112.88 & 5.58 & 112 & 347 \\
\hline $\begin{array}{l}\text { TaTPP7- } \\
\text { 3A }\end{array}$ & TraesCS3A02G085700 & $3 A$ & 55223622 & 55255982 & 1662 & 9 & 553 & 61554.27 & 8.06 & 306 & 533 \\
\hline $\begin{array}{l}\text { TaTPP7- } \\
\text { 3D }\end{array}$ & TraesCS3D02G085800 & $3 D$ & 43259299 & 43283531 & 1755 & 9 & 584 & 65022.10 & 8.86 & 337 & 564 \\
\hline $\begin{array}{l}\text { TaTPP8- } \\
5 \mathrm{~A}\end{array}$ & TraesCS5A02G190000 & $5 \mathrm{~A}$ & 394181080 & 394183400 & 1122 & 5 & 373 & 40852.40 & 8.95 & 114 & 345 \\
\hline $\begin{array}{l}\text { TaTPP8- } \\
\text { 5B }\end{array}$ & TraesCS5B02G193100 & $5 B$ & 348448002 & 348450302 & 1122 & 4 & 373 & 40926.44 & 8.97 & 114 & 345 \\
\hline $\begin{array}{l}\text { TaTPP8- } \\
\text { 5D }\end{array}$ & TraesCS5D02G200800 & $5 \mathrm{D}$ & 303758772 & 303761166 & 1122 & 5 & 373 & 40860.33 & 8.96 & 114 & 345 \\
\hline $\begin{array}{l}\text { TaTPP9- } \\
6 \mathrm{~A}\end{array}$ & TraesCS6A02G248400 & $6 \mathrm{~A}$ & 461143866 & 461147635 & 1119 & 8 & 372 & 41114.13 & 5.68 & 119 & 355 \\
\hline $\begin{array}{l}\text { TaTPP9- } \\
6 \mathrm{~B}\end{array}$ & TraesCS6B02G276300 & $6 \mathrm{~B}$ & 500209451 & 500213722 & 1119 & 8 & 372 & 41281.38 & 5.89 & 119 & 355 \\
\hline
\end{tabular}




\begin{tabular}{|c|c|c|c|c|c|c|c|c|c|c|c|}
\hline \multirow{2}{*}{$\begin{array}{l}\text { Gene } \\
\text { name } \\
\text { TaTPP9- } \\
\text { 6D }\end{array}$} & \multirow{2}{*}{$\begin{array}{l}\text { Locus_ID } \\
\text { TraesCS6D02G230500 }\end{array}$} & \multirow{2}{*}{$\begin{array}{l}\text { Chr. } \\
\text { 6D }\end{array}$} & \multicolumn{2}{|l|}{ Position (bp) } & \multirow{2}{*}{$\begin{array}{l}\text { ORF } \\
1119\end{array}$} & \multirow{2}{*}{$\begin{array}{l}\text { Introns } \\
8\end{array}$} & \multicolumn{3}{|c|}{ Deduced polypeptide } & \multicolumn{2}{|c|}{ Trehalose_Ppase } \\
\hline & & & 323712099 & 323716021 & & & 372 & 41047.03 & 5.56 & 119 & 355 \\
\hline $\begin{array}{l}\text { TaTPP10- } \\
6 \mathrm{~A}\end{array}$ & TraesCS6A02G301800 & $6 \mathrm{~A}$ & 535151913 & 535154867 & 1251 & 8 & 416 & 45367.17 & 9.26 & 114 & 341 \\
\hline $\begin{array}{l}\text { TaTPP10- } \\
6 \mathrm{~B}\end{array}$ & TraesCS6B02G330900 & $6 \mathrm{~B}$ & 581079293 & 581082545 & 1224 & 8 & 407 & 44291.96 & 8.64 & 154 & 381 \\
\hline $\begin{array}{l}\text { TaTPP10- } \\
\text { 6D }\end{array}$ & TraesCS6D02G281100 & $6 \mathrm{D}$ & 388537685 & 388540648 & 1110 & 8 & 369 & 40252.37 & 8.79 & 114 & 341 \\
\hline $\begin{array}{l}\text { TaTPP11- } \\
\text { 7A }\end{array}$ & TraesCS7A02G180800 & $7 \mathrm{~A}$ & 135006112 & 135008690 & 1086 & 9 & 361 & 39542.13 & 8.41 & 108 & 335 \\
\hline $\begin{array}{l}\text { TaTPP11- } \\
\text { 7B }\end{array}$ & TraesCS7B02G085800 & $7 B$ & 97972425 & 97975038 & 1095 & 9 & 364 & 40160.77 & 8.11 & 108 & 335 \\
\hline $\begin{array}{l}\text { TaTPP11- } \\
\text { 7D }\end{array}$ & TraesCS7D02G182600 & 7D & 136013159 & 136015620 & 1092 & 9 & 363 & 39907.51 & 8.60 & 108 & 335 \\
\hline
\end{tabular}

The phylogenetic tree of TPP proteins from wheat, maize, rice, Populus, Arabidopsis, and B. distachyum. AMEGA 6.0 was used to build a neighborjoining tree, which was visualized with the online tool Evolview. TPPs from one plant species are marked with leaf labels. I-VII denotes the seven plant TPP protein subfamilies. The percentage bootstrap scores were calculated from 1000 replications.

\section{Phylogenetic and synteny analysis of the wheat TPP gene family}

We assessed TPP genes from various plant species (with fully sequenced whole genomes) to assess the phylogenetic relationship of the TPP gene families in both wheat and other species, including monocotyledonous angiosperms maize (Zea mays), Brachypodium distachyon, and rice (Oryza sativa), and the dicotyledonous angiosperms Arabidopsis thaliana and poplar (Populus trichocarpa). We used 86 TPP proteins to produce a phylogenetic tree, which categorized the TPPs into six subfamilies (I-VII) (Fig. 1; Additional file 2: Figure S1). Subfamily II was comprised of TPP genes from six different species, while subfamilies III and IV were comprised only of TPP genes from the dicotyledonous angiosperms Arabidopsis thaliana and poplar. Subfamilies $\mathrm{V}, \mathrm{VI}$, and VII were comprised of TPP genes from monocotyledonous angiosperms maize, rice, wheat, and Brachypodium distachyon. Analysis of the phylogenetic tree indicates the presence of 4, 9, 6, 3, and 9 TaTPPs in the TPP subfamilies I, II, V, VI, and VIII, respectively (Fig. 1).

Genomic comparison is a quick and easy method of transporting genomic information from a well-studied species to a newly-studied species. We used the genomic position information to locate 31 TaTPP genes over 17 wheat chromosomes, which ranged from 1 to 5 members per chromosome (Table 1; Fig. 2). We used Holub's method [32] to identify nine tandem duplication events (TaTPP2-2A/ TaTPP3-2A, TaTPP22A/ TaTPP4-2A, TaTPP3-2A/ TaTPP4-2A, TaTPP2-2B/ TaTPP3-2B, TaTPP2-2B/ TaTPP4-2B, TaTPP3-2B/ TaTPP4-2B, TaTPP2-2D/ TaTPP3-2D, TaTPP2-2D/TaTPP4-2D, and TaTPP3-2D/TaTPP4-2D) in wheat TPP genes, suggesting that certain TaTPP genes could be produced via gene duplication (Fig. 2). Brachypodium distachyon has a close phylogenetic relationship with wheat, and is considered a model for monocotyledonous angiosperm plants. As such, we performed a synteny analysis between wheat and Brachypodium distachyon TPP genes to explore their relationship. This analysis of wheat and Brachypodium distachyon TPP genes identified 14 pairs of syntenic TPP genes between Brachypodium distachyon and wheat (with E-value $<1 \mathrm{e}^{-5}$ ), including 17 TaTPP genes (TaTPP1-1A, TaTPP1-1D, TaTPP3-2A, TaTPP3-2D, TaTPP6-2A, TaTPP6-2D, TaTPP7-3A, TaTPP7-3D, TaTPP8-5A, TaTPP8-5D, TaTPP9-6A, TaTPP9-6D, TaTPP10-6A, and TaTPP10-6D) and seven BdTPP genes (BdTPP1, $B d T P P 2, B d T P P 3, B d T P P 4, B d T P P 5, B d T P P 7$, and BdTPP8) (Fig. 2). This suggests that most TPP genes existed before Brachypodium distachyon and wheat diverged.

\section{Analysis of gene structure and motif composition}

The structural divergence of exons and introns served an important role as several families of genes have evolved [33]. We generated a different phylogenetic tree using 31 full-length wheat TaTPP protein sequences to better understand the diversity of the structure of wheat TaTPP genes. We divided the wheat TaTPP proteins into five separate subfamilies, according to the above description (Fig. 3A). We next mapped the locations of the exons/introns to the coding regions of each TaTPP gene. We found that TaTPP8-5B had four introns, TaTPP8-5A and TaTPP8-5D had five introns, TaTPP6-2D had seven introns, TaTPP5-2B had eleven introns, and TaTPP1-1B had twelve introns. Of the remaining proteins, there were 15 TaTPP genes had 8 introns and 10 TaTPPs had 9 introns (Fig. 3B). The gene structure of orthologous genes is typically highly conserved, which helps to determine their evolutionary relationships [33]. Wheat TaTPP genes in the same subfamily typically have similar gene structures (intron number and exon length), particularly those of subfamily VII, which all had eight introns (Fig. 3B). The hexaploid bread wheat genome was generated by the merging of the T. urartu (subgenome A), Aegilops speltoides (subgenome B), and A. tauschii (subgenome D) genomes hundreds of thousands of years ago. Most of the genes in the A, B, and D sub-genomes (60.1-61.3\%) have orthologs in all related diploid genomes. Analysis of the related 
intron/exon gene structures based on the phylogenetic tree provided intron gain/loss information for all TaTPP genes in the A, B, and D subgenome. Of these, four clusters altered the structure of their introns/exons, such as TaTPP1-1A/B/D, TaTPP5-2A/B, TaTPP6-2A/B/D, TaTPP8$5 A / B / D$ (Fig. 3B). Due to the high number of orthologs in the wheat $A, B$, and $D$ sub-genomes, the gain or loss of introns in these orthologs complicates the transcriptomes and proteomes found in wheat.

We next used the online MEME tool to identify the conserved motifs and assess the diverse structures of wheat TPP proteins (Fig. 4; Additional file 3: Figure S2), and found 20 conserved protein domains (with $E$-value $\leq 1 \mathrm{e}^{-30}$ ) across 31 wheat TPP proteins. The TPP domain consisted of motif 5 , $6,7,8,9$, and 10, which is a common conserved domain located in the C-terminal of all the TaTPP proteins (Additional file 4: Figure S3). Most motifs share orders within the same subfamily, and motifs with similar compositions shared by TaTPP proteins are clustered closely (Fig. 4). This suggests that those members of a particular group have similar functional characteristics.

\section{Subcellular localization of TaTPP proteins in different subfamilies}

We further characterized the subcellular localization of four TaTPPs (TaTPP6, TaTPP7, TaTPP9, TaTPP11) that belong to the distinct cluster in the phylogenetic tree shown in Fig. 1. In order to confirm the subcellular localization of these TaTPPs, we developed the 35S:: TaTPP6-GFP, 35S:: TaTPP7-GFP, 35S:: TaTPP9-GFP, and 35S:: TaTPP11-GFP transient expression vectors to express TaTPP6-GFP, TaTPP7-GFP, TaTPP9-GFP, and TaTPP11-GFP fusion proteins in wheat protoplasts, with 35S::GFP as positive control. The result was as expected, all four TaTPPs-GFP fusion proteins were located in both cytoplasm and the nucleus (Fig. 5).

\section{Cis -acting regulatory elements in TaTPP promoters}

Specific gene expression is primarily regulated by certain promoters, the action of which is mediated by transcription factors via directly binding to cis-acting regulatory elements [34]. As such, analyzing upstream regulatory sequences will contribute to a better understanding of how target genes are regulated, allowing us to assess potential functions [35]. We extracted and scanned 2000 bp of non-coding sequences upstream from the predicted translation start site of each TaTPP gene to fully identify the putative cis-acting regulatory elements. We used the online software tools PlantCARE and PlantRegMap to locate the abundant regulatory cores associated with responses to hormones, stress, and development (Fig. 6; Additional file 5: Table S2).

We observed significantly enriched hormone-related motifs in the majority of the regulatory regions of the TaTPP genes we tested, including abscisic acid (ABRE-element), auxin (TGA-element, AuxRE-core), gibberellin (P-box, GARE-motif and TATC-box), salicylic acid (TCA-element), and methyl jasmonate (TGACG- and CGTCA-motif). Statistical analysis indicated that two kinds of stress-related motifs are involved in abscisic acid and MeJA (methyl jasmonate), which were the most common cis-acting hormone-responsive elements. These elements were found in the promoters of most TaTPP genes, except TaTPP5-2B, TaTPP6-2D, TaTPP7-3D and TaTPP10-6D for ABA response and TaTPP5-2A, TaTPP7-3A, TaTPP8-5B, TaTPP8-5D, TaTPP9-6D, and TaTPP11-7D for MeJA response. Of the 31 TaTPP genes, 17 contained both gibberellin-response elements (P-box, GARE-motif and TATC-box) and auxin-response elements (TGA-element or AuxRE-core). We also found the salicylic acid-responsive TCAelement in the promoters of 11 TaTPP genes (Fig. 6; Additional file 5: Table S2).

Along with hormone-related motifs, we observed stress elements in the TaTPP gene promoters. In particular, elements pertaining to light response were found in all TaTPP gene promoters, including G-box, TCT-motif, I-box, Sp1, and MRE. Regarding drought response, seven TaTPP gene promoters possessed DRE (dehydration-responsive element) or MBS (MYB binding site involved in drought-inducibility) elements. LTR is a lowtemperature response element and is a primary component of the motifs related to stress observed in 16 TaTPPs promoters. A WUN-motif wound response element was found in 10 TaTPPgenes, while the other seven TaTPPs genes possessed TC-rich repeats, which are cis-acting elements associated with defense and stress responses. Certain cis-elements are involved in the specific expression in organs and tissues or with metabolism, including the role of MBS I in flavonoid biosynthetic genes regulation, the role of motif I in root-specific expression, the role of CAT-box in meristem expression, the role of GCN4 motif in endosperm expression, the role of the RY-element in seed-specific regulation, and the role of O2site in zein metabolism regulation, and the role of MSA-like in cell cycle regulation (Fig. 6; Additional file 5: Table S2). These results indicated that TaTPP genes might be involved in plant development, multiple hormone and stress responses.

\section{Tissue-specific expression profiles of TaTPP genes}

Gene expression is required for the normal growth and development of healthy plants, and, as a result, is highly regulated. Specific patterns of expression of candidate genes indicate potential roles in both growth and development. We used publicly available RNA-seq data to observe these expression patterns in seedling stems, seedling roots, seedling leaves, flag leaves, and during two stages of spike development ( 5 days and 15 days after head sprouting) and four stages of grain development (5 days, 10 days, 15 days, and 20 days after pollination), allowing us to assess the possible role of TaTPP genes during the growth and development of wheat. We obtained 26 TaTPP gene transcripts (Fig. 7), and could not locate five other TaTPP genes due to low levels of expression or the fact that they could be pseudogenes. Levels of expression vary widely in different tissues of wheat TaTPP genes, and between different tissues in individual TaTPPgenes. We observed three homologous genes TaTPP8-5A/B/D 
that demonstrated widespread expression patterns that were higher in almost all tissues and stages. There are high levels of TaTPP1-1A/B/D expression in both seedling stems and young spikes, while there are high levels of TaTPP2-2A and TaTPP4-2A/D expression in seedling leaves, seedling stems, and grains. Compared with the seedling roots, TaTPP3-2A/D display relatively higher expression in other tissues and stages. There are high levels of TaTPP9-6A/D expression in seedling leaves, roots, spikes, and stems. There are higher levels of TaTPP10-6A/D expression in seedling stems, leaves, and mature spikes, while there is a strong and particular expression of TaTPP2-2D, TaTPP4-2B, and TaTPP11-7A/B/D during grain development, suggesting that these genes could play significant roles during this stage (Fig. 7).

Most homologous genes demonstrate similar patterns of expression during developmental stages, though several clustered expression profiles do not have similar genes, including copies of individual kinds of TaTPP genes from their sub-genomes; some demonstrate opposite expression patterns. For example, TaTPP2-2A is found on chromosome $2 \mathrm{~A}$ and is preferentially expressed in the seedling leaves and stems, while the homologous TaTPP2-2D gene (located on chromosome 2D) is expressed in these tissues at a lower point. TaTPP10-6A is located on 6A and displays higher levels of expression in mature spikes and seedling stems. The homologous TaTPP10-6B, found on 6B, is expressed preferentially in the seedling leaves, stems, and mature spikes, while homologous genes from 6D are expressed only in the seedling leaves (Fig. 7). This difference in expression profiles between homologous genes from different subgenomes demonstrates that some have acquired new functions or lost old functions following polyploidization during wheat's evolutionary history.

\section{Expression analysis of TaTPP genes respond to abiotic stresses}

Environmental stresses significantly affect the productivity of wheat, making it important to study the wheat genes responsible for stress response in order to increase yields. We used quantitative real-time PCR (qRT-PCR) to assess how TaTPP gene expression responds to continuous ABA, low temperature, and salt stress, allowing us to analyze the role of TaTPP genes that could be associated with plant defense to abiotic stresses. We designed allele pairs from A-, B- and D-subgenomes and tested them together, since the products of their transcription share similar sequences. Each gene we analyzed had a different expression when responding to a minimum of one abiotic stress (Fig. 8). In response to ABA, there were eight up-regulated TaTPPs (TaTPP1, TaTPP3, TaTPP4, TaTPP6, TaTPP7, TaTPP8, TaTPP9, and TaTPP11) and three down-regulated TaTPPs (TaTPP2, TaTPP5, and TaTPP10) in seedling leaves at a minimum of one time point. In response to low-temperature conditions, there were seven up-regulated TaTPPs (TaTPP1, TaTPP3, TaTPP4, TaTPP7, TaTPP8, TaTPP9, and TaTPP11) and four down-regulated TaTPPS (TaTPP2, TaTPP5, TaTPP6, and TaTPP10). In response to salt stress, there were eight up-regulated TaTPPs (TaTPP1, TaTPP3, TaTPP4, TaTPP6, TaTPP7, TaTPP8, TaTPP9, and TaTPP11) and three down-regulated TaTPPs (TaTPP2, TaTPP5, and TaTPP10) (Fig. 8).

To better understand the functions of TaTPP genes in regulating wheat drought response, the expression patterns of 11 TaTPPs were experimentally examined in leaves and roots of 3-week-old drought-stressed wheat seedlings. As illustrated in Fig. 9, a dramatic upregulation of 8 TaTPP genes (TaTPP1, TaTPP2, TaTPP3, TaTPP4, TaTPP5, TaTPP9, TaTPP10, and TaTPP11) were observed in response to drought stress, especially in the leaves. TaTPP7 also showed a slightly up-regulation in leaves and roots after drought stress. The changed expression levels of TaTPP2, TaTPP3 and TaTPP4 in leaves after drought stress were very sharp, with more than 60 folds, indicating that these genes are extremely susceptible to drought stress. Some genes showed very similar expression profiles after drought stress, such as TaTPP3 and TaTPP4 pairs. Some TaTPPs were significantly upregulated after light/early drought stress, such as TaTPP2, TaTPP3, TaTPP4 and TaTPP9 in leaves and TaTPP9 and TaTPP11 in roots, suggesting positive roles of these genes in early drought stress response. Some genes were significantly upregulated after severe stress, such as TaTPP1, TaTPP10 and TaTPP11 in leaves and TaTPP6 and TaTPP8 in roots, suggesting that these genes are important for the plant response to drought stress at a severe level. Upon drought stress, TaTPP5 and TaTPP10 were upregulated in leaves but downregulated in roots (Fig. 9). These data show the potential of some TaTPP genes for enhancing adversity resistant capacity, especially for wheat drought improvement.

\section{Ectopic expression of TaTPP11 in Arabidopsis delayed plant development and enhanced drought tolerance}

Alignment of the protein sequences determined the presence of three TaTPP11 homeologs sharing a sequence similarity of approximately $95 \%$ (Additional file 6: Figure S4). Additional information regarding the spatiotemporal profile of TaTPP11 expression could contribute to a better understanding of how TaTPP11 functions biologically. In this case, we observed TaTPP11 expression across various tissues and organs of wheat at different stages of development, such as the roots and leaves of seedlings, young panicles, flag leaves, and seeds. Our results demonstrated high levels of TaTPP11 expression in seedling leaves and developing seeds, and low levels of TaTPP11 expression in developing panicles (Fig. 10A). This indicates that TaTPP11 could serve an important purpose as wheat seeds develop.

To better understand how TaTPP11 relates to the development of wheat, we produced 35S:: TaTPP11-7D transgenic Arabidopsis lines and assessed their levels of TaTPP11-7D expression to select three independent transgenic lines (OE1, OE2, and OE3) for subsequent analysis (Fig. 10B; Additional file 7: Figure S5). First, we observed the germination of the seeds and found there were no significant differences between transgenic and wild type lines (Fig. 10C, D). Next, we detected the phenotypes of the 35S:: TaTPP11-7D transgenic Arabidopsis lines and that of the wild-type over 
various developmental stages. The TaTPP11-7D transgenic Arabidopsis seedlings grew vegetation for much longer, bolted and flowered later, and had a lower plant height compared to the wild type (Fig. 10E). We also analyzed the organs from both the Arabidopsis wild-type plants and transgenic lines, and did not find any significant differences in the organs of the transgenic and wild type varieties, including in the seeds, flowers, and siliques (Fig. 10E, F).

We next assessed the drought tolerance of the wild type and transgenic plants to identify the role of TaTPP11 in plant drought stress. Both 35S:: TaTPP11-7D transgenic plants and wild type plants were grown for three weeks in soil, after which they were not watered for 14 days. The plants were then watered for six days. Afterward, approximately $30 \%$ of wild type plants survived, while approximately $80-90 \%$ of the transgenic plants survived (Fig. 11A, B). Next, the trehalose contents of the wild type plants and the 35S:: TaTPP11-7D transgenic plants were assayed, demonstrating that the trehalose levels in transgenic lines were significantly higher than in wild type plants (Fig. 11C). Additionally, we assessed other cellular processes impacted by drought stress conditions, paying particular attention to substances regulating osmosis. We observed increased levels of proline and soluble sugar, and decreased levels of malonaldehyde under drought stress conditions in transgenic lines (Fig. 11DI). These results indicate that TaTPP11 overexpression in Arabidopsis might delay the development of plants, but could also increase the drought tolerance of transgenic varieties.

\section{Discussion}

There is a significant body of evidence indicating that T6P is an important signal metabolite in plants that affects metabolism, growth, and development. Therefore, there is a pressing need to better understand the processes and function of several plant TPP proteins. Whole-genome sequencing and the widespread nature of worldwide genomic databases have allowed researchers to closely analyze complex genomes, such as wheat [35]. Identifying TPP genes in wheat is needed to additionally characterize these genes. While the family of TPP genes has been studied in both monocots and dicots, their specific functions are still unclear, particularly in wheat. To better understand this function, we comprehensively identified and analyzed TPP genes in wheat.

We identified 31 candidate genes in the family of TPP genes in wheat (Table 1). There were three times as many TPPs in wheat as in Brachypodium distachyon and rice, which is evidenced by the fact that hexaploid wheat descended from a hybridization of A-, B-, and D-genome ancestors approximately one or two million years ago $[37,38]$. This study assessed certain traits found in wheat TPP genes. The majority of TaTPP genes encode proteins with a predicted molecular mass of 39-65 kDa (with the exception of TaTPP5-2A) as well as an isoelectric point of approximately 5.5-9.2, which is similar to the TPPs found in other species of plants, while the majority of TaTPP genes possessed 8-9 introns (Table 1). We performed multiple sequence alignment and analyzed the phylogenetics of the 31 TaTPP protein sequences. We observed significant sequence divergence, particularly at the C-terminus, during the multiple sequence alignment of wheat TaTPP proteins (Additional file 4: Figure S3). This demonstrates the variable role that TaTPP genes serve during the growth and development of plants [2,30]. We classified the wheat TaTPP protein family into five subfamilies (I, II, V, VI, VII) by assessing how they relate to homologous TPPs in other species (Fig. 1; Fig. 3A), gene structures (Fig. 3), and motif arrangements (Fig. 4). The smallest subfamily was the TaTPP VI subfamily (Fig. 1; Fig. 3A). Analysis of the gene structure demonstrated that genes from each TaTPP subfamily have similar numbers and positions of their exon-intron structures (Fig. 3B), though the TaTPP I subfamily diverged more (Fig. 3B). This suggests that the TaTPP I subfamily genes could perform different roles during the development of wheat. Additionally, wheat TaTPP proteins all possess particular conserved TPP domains, consisting of six conserved motifs (Fig. 4). With the exception of TPP domain-based conserved motifs, there are specific motifs for each TaTPP subfamily. For example, motifs 1 and 2 exist only in TaTPP VII subfamily proteins, while motif 11 only exists in the TaTPP VI subfamily. Motifs 18,19 , and 20 only exist in the TATPP I subfamily (Fig. 4).

Duplicate genes located on different chromosomes are considered segregation duplication events. It is common for gene duplication events to occur in wheat, which will assist in the future analysis of the function and evolution of genes [31]. Whole genome duplications are common in angiosperms [39], and typically expand the gene family [40]. Gene duplication can result in the emergence of novel functions for certain plant genes. Syntenic relations and segregation duplication events between Brachypodium distachyon and wheat suggest that certain TaTPP genes were produced via gene duplication, indicating their similar origins (Fig. 2).

We analyzed the expression of publicly available RNA-seq data from 10 organs/tissues at various stages of development to assess the role play by TPP genes across wheat's life cycle. Wheat TaTPP genes are similar to Arabidopsis in that they are differentially transcribed, depending on the tissue, stage, and cell [23]. High levels of TaTPP genes expression were detected in the leaves, such as TaTPP2-2A, TaTPP3-2A/D, TaTPP4-2A/D, TaTPP8-5A/B/D, TaTPP9-6A/D and TaTPP10-6B/D (Fig. 7). This is similar to prior results, which found higher levels of expression of TPPA, TPPB, and TPPG in the leaves compared to other organs [23]. There were higher levels of TaTPP9-6A/D and TaTPP8-5A/B/D expression in the roots, which is similar to TPPA, while there were higher levels of TPPD, TPPG, and TPP/ in the root caps and protoderms [23], indicating that they could be involved in the development of roots. In wheat, TaTPP2-2A/D, TaTPP3-2A/D, TaTPP4-2A/B/D, TaTPP8-5A/B/D, and TaTPP11-7A/B/D all demonstrated higher levels of expression in the grains at various stages of development, indicating they could be involved in grain development (Fig. 7). These results indicate that TaTPP genes could serve a variety of roles during the development of wheat. Additional ectopic TaTPP11 expression in Arabidopsis displayed delays in development and flowering (Fig. 10), highlighting the importance of TaTPP11 during plant development. 
The expression or transcription of genes begins with an upstream regulatory promoter region, which is the combination of several cis-acting regulatory components joined with a minimal basic start element. Different regulatory cores provide the promoters with strength, time-space specificity, and stimuli response. As such, analyzing a target gene promoter's regulatory elements can allow us to predict how its expression will respond to different stimuli. A review of the promoters of the TaTPP gene family demonstrated that several phytohormone-, stress- and development-related regulatory elements were present (Fig. 6; Additional file 5: Table S2). Significant amounts of hormone-related cis-acting regulatory elements in most TaTPP promoters suggest that they play important roles as regulators of the core in various hormone-signaling pathways. Members of the TaTPP gene family play roles in response to biotic and abiotic stresses, which is consistent with enriching several ciselements related to stress. Certain TaTPP promoters possess regulatory cores related to stress, such as ABA-responsive elements ABRE, SAresponsive elements (TCA-elements), defense and stress-responsive elements (TC-rich repeats and MBS), low-temperature-related elements (LTR), MeJA-responsive elements (CGTCA- and TGACG-motif), and wound-responsive elements (WUN-motif) (Additional file 5: Table S2). Of these, CGTCAand TGACG-motifs are typical MeJA-responsive cis-acting elements, and were found in 20 of 31 promoters of the TaTPP genes. Jasmonic acid, a primary growth hormone, regulates resistance to plant diseases and responses to abiotic stresses [41]. We also observed certain elements specific to organs or tissues related to the development of roots, seeds, or endosperms (Fig. 6; Additional file 5: Table S2). Promoters of TaTPP2-2B, TaTPP4-2D, TaTPP7-3A, TaTPP8-5A, TaTPP8-5B, and TaTPP10-6B possess RY-element, a seed-specific regulation core, which indicates that they could play a role in seed development. The TaTPP6-2A and TaTPP6-2B genes should be further studied, since they possess the root-specific motif I element and could play a role in the development of roots (Fig. 6; Additional file 5: Table S2).

Abiotic stresses are primarily responsible for reducing crop quality and yield. Recent research has sought to better understand how plants respond to abiotic stresses, which would contribute to improved crop tolerance. While the overexpression of E. coli. TPS and TPP fusion proteins can increase the tolerance of abiotic stress in rice [3], there is little known about how endogenous TPP functions in plants. We analyzed the cis-acting regulatory elements and found high levels of regulatory cores in the TaTPP gene family promoters. We performed qRT-PCR analysis to observe how TaTPP genes found in the seedling leaves respond to salt, low-temperature, ABA, and drought stresses (Fig. 8; Fig. 9). Our results demonstrated that the majority of TaTPP genes were up-regulated after ABA treatment, while some decreased. We saw the same expression profiles after drought, salt, and low-temperature treatments, however, the intensity of induction and the whether the expression was suppressed or up-regulated was different between all stressors. All four abiotic stresses regulated TaTPP gene expression positively and negatively in wheat (Fig. 8; Fig. 9), though the leaves and roots responded differently to drought stress conditions (Fig. 9). The fact that TaTPP2, TaTPP5, and TaTPP10 were all down-regulated under low-temperature, ABA, and salt stress treatments suggests they are suitable candidates for using CRISPR-Cas9 gene editing to improve the abiotic tolerance of wheat plants. TaTPP genes respond to stress in a manner that indicates they assist in adapting to variable environmental conditions.

\section{Conclusions}

We identified 31 TPP family genes in wheat, all of which have at least one conserved TPP domain. The TPP family in wheat can be classified into five subfamilies based on their similar exon/intron structures and motifs. Additionally, there are four TaTPP protein found in the cytoplasm and nucleus (TaTPP6, TaTPP7, TaTPP9, and TaTPP11). A pattern analysis of tissue-specific expression demonstrated that TaTPP genes in wheat are expressed differentially, indicating that they play different roles in the growth and development of wheat. Analysis of the promoter cis-elements and expression patterns when subjected to abiotic and biotic stresses demonstrate that TaTPP genes in wheat respond to different stimuli. Additionally, the ectopic expression of TaTPP11 in Arabidopsis demonstrated that plant development is delayed and drought tolerance is enhanced, highlighting the importance of TaTPP11 during both plant development and in response to stress. This study provides significant information on the wheat TPP gene family, which will allow for the future study of their functional divergence and how they can be manipulated in the future.

\section{Methods}

\section{Stress treatment and plant material}

We used the wheat cultivar Chinese spring for this study, which was acquired from Northwest A\&F University. However, we also could have obtained this variety from Chinese Crop Germplasm Resources Information System (http://www.cgris.net/zhongzhidinggou/index.php). The cultivar was surface-sterilized using $75 \%$ ethanol, after which it was washed using deionized water and germinated on wet filter paper for three days at $25^{\circ} \mathrm{C}$. We then placed the germinated seeds in a nutrient solution $\left(0.1 \mathrm{mM} \mathrm{KCl}, 0.75 \mathrm{mM} \mathrm{K}_{2} \mathrm{SO}_{4}, 0.65 \mathrm{mM} \mathrm{MgSO}_{4}, 0.25 \mathrm{mM} \mathrm{KH}_{2} \mathrm{PO}_{4}, 1.0 \mathrm{mM} \mathrm{MnSO}_{4}, 1.0 \mathrm{mM}\right.$ $\mathrm{ZnSO}_{4}, 0.1 \mathrm{mM}$ EDTA-Fe, $\left.2.0 \mathrm{mM} \mathrm{Ca}\left(\mathrm{NO}_{3}\right)_{2}, 0.005 \mathrm{mM}\left(\mathrm{NH}_{4}\right)_{6} \mathrm{Mo}_{7} \mathrm{O}_{24}, 0.1 \mathrm{mM} \mathrm{CuSO}{ }_{4}\right)$ and hydroponically cultivated them in a $16^{\circ} \mathrm{C}$ growth chamber under a 16/8 h light/dark cycle.

For $\mathrm{ABA}$ and salt treatments, we immersed seedlings at the three-leaf stage into hydroponic solutions with $200 \mathrm{mmol} \mathrm{NaCl}$ and $100 \mu \mathrm{mol} \mathrm{ABA}$, and obtained samples at $0,1,3,6,12$, and $24 \mathrm{~h}$ after treatment. For low-temperature treatments, we immersed seedlings at the three-leaf stage into hydroponic solutions at $4^{\circ} \mathrm{C}$ for $0,1,3,6,12$, and $24 \mathrm{~h}$ and obtained samples. For drought treatment, we placed seedlings at the three-leaf stage onto a clean bench and subjected them to drought conditions $\left(25^{\circ} \mathrm{C}\right.$, relative humidity $\left.40-60 \%\right)$ and collected the roots and leaves from three seedlings at $0,1,3,6,12$ and $24 \mathrm{~h}$. We quickly froze all samples in liquid nitrogen and stored them at $-80^{\circ} \mathrm{C}$ to isolate the RNA. 


\section{Quantitative real-time PCR and RNA extraction}

We used a Total RNA Rapid Extraction Kit for Polysaccharides Polyphenol Plant (BioTeke), according to the instructions of the manufacturer, to isolate and purify the total RNA. We then treated the resulting purified RNA with RNase-free DNase I (TaKaRa, China) to remove traces of DNA and ensure the sample was not contaminated. We then synthesized first-strand cDNA from $1 \mu \mathrm{g}$ of total RNA with Recombinant M-MLV reverse transcriptase (Promega, USA) and used an ABI7300 Thermo-cycler (Applied Biosystems, USA) to conduct quantitative real time-PCR (qRT-PCR) in optical 96-well plates. All reactions were performed in $10 \mu$ l volume, with $1 \mu$ l diluted cDNA, 200 nM gene-specific primers, and $5 \mu$ l SYBR Premix Ex Taq II (TaKaRa) according to the following: $10 \mathrm{~min}$ at $95^{\circ} \mathrm{C}$, and then 40 cycles of $15 \mathrm{~s}$ at $95^{\circ} \mathrm{C}$ and $30 \mathrm{~s}$ at $60^{\circ} \mathrm{C}$. We verified the specificity of each primer's amplicon via melting curve analysis and used the wheat Actin (Gene ID: 542814) as an internal control for analyzing the expression of TaTPP11 in wheat. We calculated relative levels of gene expression levels using the $2^{-\triangle \triangle C t}$ method [42], while expression variation was estimated from three biological replicates. Additional file 6: Table S3 outlines the primer pairs used in qRT-PCR analysis.

\section{Genome-wide identification and annotation of TPP genes in wheat}

We used 23 TPP protein sequences from Arabidopsis and rice to identify the genes from the Chinese Spring IWGSC RefSeq v1.1 reference genome assembly (Ensembl Plants; https://plants.ensembl.org/Triticum_aestivum/Info/Index) with the local blast program (E-value < 1e-10). After removing duplicate searches using the CD-hit program, we identified the rest of the protein sequences with the Simple Modular Architecture Research Tool (SMART; http://smart.embl-heidelberg.de/smart/set_mode.cgi?NORMAL=1). We performed a phylogenetic analysis to filter the genes from TPP proteins previously identified from Brachypodium distachyon, Populus trichocarpa (poplar), Arabidopsis thaliana, Oryza sativa (rice), and Zea mays (maize). We also used phylogenetic analysis to sort the various TPP subfamilies and named the TPP genes from each of three wheat subgenomes ( $A, B$, and $D$ genomes) TaTPPX_ZA, TaTPPX_ZB, or TaTPPX_ZD, respectively, where $X$ is the gene number and $Z$ indicates its location on the wheat chromosome. We calculated the theoretical pl (isoelectric point) and Mw (molecular weight) of each putative wheat TPP protein using ExPasy (http://web.expasy.org/compute_pi/).

\section{Systematic analysis of the bioinformatics of the wheat TPP family}

In order to perform a phylogenetic analysis of the wheat TPP family along with other species of plants, we obtained proteomes of Populus trichocarpa (poplar), Brachypodium distachyon, Arabidopsis thaliana, Zea mays (maize), and sativa (rice) from JGI (https://phytozome.jgi.doe. gov/pz/portal.html). We acquired all of the TPP protein sequences either directly from the supplemental materials or from the proteomes based on the gene locus, as indicated by other papers. We used the ClustalW program (default settings) to produce the multiple sequence alignments [43], and produced unrooted phylogenetic trees using the neighbor-joining (NJ) method and the MEGA6.0 software, using the full-length of the TPP protein sequences [44]. We used 10,000 replications to estimate the bootstrap probability of each branch. We acquired information on the gene structure of TaTPP genes from the Chinese Spring IWGSC RefSeq v1.1 reference genome, which were analyzed with the Gene Structure Display Server 2.0 (GSDS; http://gsds.cbi.pku.edu.cn/). We identified the conserved TaTPPs motifs with the online MEME program (http://meme-suite.org/), while we used the circlize package in $\mathrm{R}$ to assess the chromosomal distribution and draw the collinearity map. We analyzed the cis-acting regulatory elements using plantCARE (http://bioinformatics.psb.ugent.be/webtools/plantcare/html/).

\section{Using RNA-seq data to analyze gene expression}

We obtained RNA-seq data from ten different tissues, including the leaves, roots, and stems of five-leaf stage wheat seedlings, spikes at the heading stage, young spikes at early booting stage, flag leaves at the heading stage, and the grains of 5, 10, 15 and 20 DPA in order to analyze how TaTPP genes were expressed in different tissues (http://genedenovoweb.ticp.net:81/Wheat_GDR1246/index.php?m=index\&f=index). We used Cufflinks and TopHat to assess gene expression, based on the RNA-seq data $[45,46]$ and calculated the FPKM value (fragments per kilobase of transcript per million fragments mapped) for each TaTPP gene. To produce the heat map, we used the $\log _{10}$-transformed (FPKM +1) values of the TaTPP genes.

\section{Subcellular localization}

We generated green fluorescent protein (GFP) expression vectors (CaMV35S-GFP-NOS) to analyze the subcellular localization of the TaTPP proteins. PCR and gene-specific primers were used to amplify the coding regions of TaTPP6, TaTPP7, TaTPP9, and TaTPP11, which were independently connected to the N-terminus of GFP in the expression vector. We isolated the wheat protoplasts from the mesophyll tissue of 2-weekold wheat seedlings, and used the PEG transfection method, along with the plasmid DNA of 35S::TaTPP6-GFP, 35S::TaTPP7-GFP, 35S::TaTPP9GFP, 35S::TaTPP11-GFP, and 35S::GFP control, as described previously, for transformation [47]. Following PEG transfection, we incubated the wheat protoplasts in W5 solution ( $2 \mathrm{mM} \mathrm{MES}, 154 \mathrm{mM} \mathrm{NaCl}, 125 \mathrm{mM} \mathrm{CaCl}$, and $5 \mathrm{mM} \mathrm{KCl}, \mathrm{pH}=5.7$ ) in a dark chamber for $18 \mathrm{~h}$ at $23^{\circ} \mathrm{C}$, and observed GFP fluorescence using a laser-scanning confocal microscope (A1, Nikon Corporation, Tokyo, Japan). 


\section{Arabidopsis transformation and TaTPP11 isolation}

We obtained the Arabidopsis ecotype Columbia from Professor Zhensheng Kang's Lab (Northwest A\&F University, China), which we used to transform TaTPP11. The full-length opening reading frame of TaTPP11 was amplified from the wheat variety Chinese Spring with gene-specific primers that were closed using the cauliflower mosaic virus (CaMV) 35S promoter, into the pGreen0029-GFP vector. The recombinant vector (35S::TaTPP11-7D) was then introduced into Agrobacterium tumefaciens, after which the floral dip method [48] was used to turn it into Arabidopsis (Arabidopsis thaliana; ecotype Columbia). We placed the $\mathrm{T}_{1}$ seeds in an MS medium with $2 \%$ sucrose and $50 \mathrm{mg} / \mathrm{mL}$ kanamycin, allowing us to identify the transformants. Homozygous $T_{3}$ plants were used to analyze the phenotype.

\section{Drought tolerance assay}

Seven-day-old seedlings from the WT and 35S::TaTPP11-7D transgenic lines were transferred from the MS medium to pot that had 230 $\mathrm{g}$ of a 2:1 ratio of nutrient soil and vermiculite (with 16 plants in each pot) for the drought tolerance assays. The 21-day-old plants that had been grown in a suitable environment (a relative humidity of $60 \%$, a photoperiod of $16-\mathrm{h} / 8$-h light/dark, and a temperature of $22^{\circ} \mathrm{C}$ ) were then exposed to drought stress conditions, where we did not water the plants for approximately 14 days. They were then watered and allowed to recover. We observed the number of plants that survived six days later, and compared a minimum of 48 transgenic plants with WT plants in each data. We performed four independent experiments and used the data from each for our statistical analysis, while a Student's $t$-test was used to assess the differences between the WT and transgenic plants.

\section{Phylogenetic analysis and multiple sequence alignments}

A phylogenetic analysis was performed on the full-length protein sequences from the TPP proteins of different plant species. We used MEGA (v6.0) software and the Neighbor-Joining ( $\mathrm{NJ}$ ) algorithm with 1,000 bootstrap re-samplings to construct the phylogenetic tree, while the ClustalW software was used to conduct multiple sequence alignments, which were manually edited with BioEdit (v7.1).

\section{Statistical analyses}

Each experiment was performed in triplicate, and data is presented and was analyzed after calculating the mean \pm standard deviation (SD) of each experiment. A Student's $t$-test was used to assess the statistical differences, while $P<0.05$ was considered statistically significant and $P<0.01$ was considered extremely statistically significant.

\section{Abbreviations}

ABA: Abscisic acid; BLAST: Basic local alignment search tool; CDS: Coding sequences; DRE: dehydration-responsive element; GSDS: Gene Structure Display Server; LTR: low temperature responsive element; MBS: MYB binding site involved in drought-inducibility; MEME: Multiple Expectation Maximization for Motif Elicitation; MYB: MYB recognition site; MYC: MYC recognition site; Pi: isoelectricpoint; SMART: Simple Modular Architecture Research Tool.

\section{Declarations}

\section{Ethics approval and consent to participate}

Not applicable.

\section{Consent for publication}

Not applicable.

\section{Availability of data and material}

All data generated or analyzed during this study are included in this article and its supplementary information files. However, the sequence data in this study can also be accessed at http://genedenovoweb.ticp.net:81/Wheat_GDR1246/index.php?m=index\&f=index. In addition, al 
Chinese Crop Germplasm Resources Information System: http://www.cgris.net/zhongzhidinggou/index.php

Ensembl Plants: https://plants.ensembl.org/Triticum_aestivum/Info/Index

ExPasy: http://web.expasy.org/compute_pi/

GSDS: http://gsds.cbi.pku.edu.cn/

JGI: https://phytozome.jgi.doe.gov/pz/portal.html

MEME: http://meme-suite.org/

plantCARE: http://bioinformatics.psb.ugent.be/webtools/plantcare/html/

SMART: http://smart.embl-heidelberg.de/smart/set_mode.cgi?NORMAL=1

\section{Competing interests}

The authors declare that they have no conflict of interest.

\section{Funding}

Grants from the Natural Science Basic Research Plan in Shaanxi Province of China (grant no. 2019JCW-18), the National Natural Science Foundation of China (grant no. 32072002), and Shaanxi Innovation Team Project (grant no. 2018TD-004) all supported the work on this project. These funding bodies had no role in the design of this study, collection, analysis, and interpretation of data or in writing the manuscript.

\section{Authors' contributions}

Z.K. and H.M. conceived and initiated the research; H.M. designed the experiments; L.D., S.L., L.D. and X.C. performed the experiments. H.M. analyzed the data and wrote the manuscript. All authors have read and approved the final manuscript.

\section{Acknowledgements}

We would like to thank all reviewers for carefully reading our manuscript, and all editors for carefully editing the manuscript. We also thank the members of the Bioinformatics Center of Northwest A \& F University for their helpful feedback.

\section{References}

1. Paul MJ, Gonzalez-Uriarte A, Griffiths CA, Hassani-Pak K. The role of trehalose 6-phosphate in crop yield and resilience. Plant Physiol. 2018;177(1):12-23.

2. Fichtner F, Lunn JE. The role of trehalose 6-phosphate (Tre6P) in plant metabolism and development. Annu Rev Plant Biol. 2021;72:737-760.

3. Garg AK, Kim JK, Owens TG, Ranwala AP, Choi YD, Kochian LV, et al. Trehalose accumulation in rice plants confers high tolerance levels to different abiotic stresses. Proc Natl Acad Sci USA. 2002;99(25):15898-15903.

4. Paul MJ, Primavesi LF, Jhurreea D, Zhang Y. Trehalose metabolism and signaling. Annu Rev Plant Biol. 2008;59(1):417-441.

5. Mu M, Lu XK, Wang JJ, Wang DL, Yin ZJ, Wang S, et al. Genome-wide identification and analysis of the stress-resistance function of the TPS (Trehalose-6-Phosphate Synthase) gene family in cotton. BMC Genet. 2016;17:54.

6. Yadav UP, Ivakov A, Feil R, Duan GY, Walther D, Giavalisco P, et al. The sucrose-trehalose 6-phosphate (Tre6P) nexus: specificity and mechanisms of sucrose signalling by Tre6P. J Exp Bot. 2014;65(4):1051-1068.

7. Astrid W. The function of trehalose biosynthesis in plants. Phytochemistry. 2002;60(5):437-440.

8. Zeid IM. Trehalose as osmoprotectant for maize under salinity-induced stress. Research Journal Agriculture Biology Science. 2009;5(5):613622.

9. Ali Q, Ashraf M. Induction of drought tolerance in maize (Zea mays L.) due to exogenous application of trehalose: growth, photosynthesis, water relations and oxidative defence mechanism. J Agron Crop Sci. 2011;197(4):258-271.

10. Julieta RS, Ramón S, Jesús C, Gabriel I. Trehalose accumulation in Azospirillum brasilense improves drought tolerance and biomass in maize plants. Fems Microbiology Lett. 2010;296(1):52-59. 
11. Satoh-Nagasawa N, Nagasawa N, Malcomber S, Sakai H, Jackson D. A trehalose metabolic enzyme controls inflorescence architecture in maize. Nature. 2006;441(7090):227-230.

12. Carillo P, Feil R, Gibon Y, Satoh-Nagasawa N. A fluorometric assay for trehalose in the picomole range. Plant Methods. 2013;9(1):21-21.

13. Nuccio ML, Wu J, Mowers R, Zhou HP, Meghji M, Primavesi LF, et al. Expression of trehalose-6-phosphate phosphatase in maize ears improves yield in well-watered and drought conditions. Nat Biotechnol. 2015;33(8):862-869

14. Figueroa CM, Lunn JE. A tale of two sugars: trehalose 6-phosphate and sucrose. Plant Physiol. 2016;172(1):7-27.

15. Griffiths CA, Sagar R, Geng Y, Primavesi LF, Patel MK, Passarelli MK, et al. Chemical intervention in plant sugar signalling increases yield and resilience. Nature. 2016;540(7634):574-578.

16. Wahl V, Ponnu J, Schlereth A, Arrivault S, Langenecker T, Franke A, et al. Regulation of flowering by trehalose-6-phosphate signaling in Arabidopsis thaliana. Science. 2013;339(6120):704-707.

17. Hulsmans S, Rodriguez M, Coninck BD, Rolland F. The SnRK1 energy sensor in plant biotic interactions. Trends Plant Sci. 2016;21(8):648-661.

18. Cabib E, Leloir LF. The biosynthesis of trehalose phosphate. J Biol Chem. 1958;231(1):259-259.

19. Avonce N, Mendoza-Vargas A, Morett E, Iturriaga G. Insights on the evolution of trehalose biosynthesis. BMC Evol Biol. 2006;6(1):109.

20. Lunn JE. Gene families and evolution of trehalose metabolism in plants. Funct Plant Biol. 2007;34(6):550-563.

21. Dijck PV. The Cytophaga hutchinsonii ChTPSP: First characterized bifunctional TPS-TPP protein as putative ancestor of all eukaryotic trehalose biosynthesis proteins. Mol Biol Evol. 2010;27(2):359-369.

22. Yang HL, Liu YJ, Wang CL, Zeng QY, Natarajan K. molecular evolution of trehalose-6-phosphate synthase (TPS) gene family in populus, Arabidopsis and rice. Plos One. 2012;7(8):e42438.

23. Vandesteene L, Lopez-Galvis L, Vanneste K, Feil R, Maere S, Lammens W, et al. Expansive evolution of the trehalose-6-phosphate phosphatase gene family in Arabidopsis. Plant Physiol. 2012;160(2):884-896.

24. Krasensky J, Broyart C, Rabanal FA, Jonak C. The redox-sensitive chloroplast trehalose-6-phosphate phosphatase AtTPPD pegulates salt stress tolerance. Antioxid Redox Signal. 2014;21(9):1289-1304.

25. Lin Q, Yang J, Wang Q, Zhu H, Wang K. Overexpression of the trehalose-6-phosphate phosphatase family gene AtTPPF improves the drought tolerance of Arabidopsis thaliana. BMC Plant Biol. 2019;19(1):381-381.

26. Lin Q, Wang S, Yihang D, Wang J, Wang K. Arabidopsis thaliana trehalose-6-phosphate phosphatase gene TPPI enhances drought tolerance by regulating stomatal apertures. J Exp Bot. 2020;71(14):4285-4297.

27. Pramanik M, Imai R. Functional identification of a trehalose 6-phosphate phosphatase gene that is involved in transient induction of trehalose biosynthesis during chilling stress in rice. Plant Mol Biol. 2005;58(6):751-762.

28. Shima, Matsui, Tahara, Imai. Biochemical characterization of rice trehalose-6-phosphate phosphatases supports distinctive functions of these plant enzymes. FEBS J. 2007;274(5):1192-1201.

29. Kretzschmar T, Pelayo M, Trijatmiko KR, Gabun Ad A L, Alam R, Jimenez R, et al. A trehalose-6-phosphate phosphatase enhances anaerobic germination tolerance in rice. Nat Plants. 2015;1(9):15124.

30. Claeys H, Vi SL, Xu X, Satoh-Nagasawa N, Eveland AL, Goldshmidt A, et al. Control of meristem determinacy by trehalose 6-phosphate phosphatases is uncoupled from enzymatic activity. Nat Plants. 2019;5(4):352-357.

31. Appels R, Eversole K, Feuillet C, Keller B, Rogers J, Stein N, et al. Shifting the limits in wheat research and breeding using a fully annotated reference genome. Science. 2018;361(6403):eaar7191.

32. Holub EB. The arms race is ancient history in Arabidopsis, the wildflower. Nat Rev Genet. 2001;2(7):516-527.

33. Xu G, Guo C, Shan H, Kong H. Divergence of duplicate genes in exon-intron structure. Pro Nat Acad Sci USA. 2012;109(4):1187-1192.

34. Lescot, M. PlantCARE, a database of plant cis-acting regulatory elements and a portal to tools for in silico analysis of promoter sequences. Nucleic Acids Res. 2002;30(1):325-327.

35. Yamaguchi-Shinozaki K, Shinozaki K. Organization of cis-acting regulatory elements in osmotic- and cold-stress-responsive promoters. Trends Plant Sci. 2005;10(2):88-94.

36. Li F, Mei F, Zhang Y, Li S, Mao H. Genome-wide analysis of the AREB/ABF gene lineage in land plants and functional analysis of TaABF3 in Arabidopsis. BMC Plant Biol. 2020;20(1):558.

37. Feldman M, Levy AA. Allopolyploidy - a shaping force in the evolution of wheat genomes. Cytogenet Genome Res. 2005;109(1-3):250-258.

38. Marcussen T, Sandve SR, Heier L, Spannagl M, Pfeifer M, Jakobsen KS, et al. Ancient hybridizations among the ancestral genomes of bread wheat. Science. 2014;345(6194):1250092.

39. Flagel LE, Wendel JF. Gene duplication and evolutionary novelty in plants. New Phytol. 2009;183(3):557-564.

40. Li Z, Zhang C, Guo Y, Niu W, Wang Y, Xu Y. Evolution and expression analysis reveal thepotential role of the HD-Zip gene family in regulation of embryo abortion in grapes (Vitis vinifera L.). BMC genomics. 2017;18(1):744.

41. Ruan J, Zhou Y, Zhou M, Yan J, Khurshid M, Weng W, et al. Jasmonic acid signaling pathway in plants. Int J Mol Sci. 2019;20(10):2479.

Page 13/24 
42. A KJL, B TDS. Analysis of Relative gene expression data using real-time quantitative PCR and the 2(-Delta Delta C(T)) Method. Methods. 2001;25(4):402-408

43. Thompson J, Gibson T, Higgins D. Multiple sequence alignment using ClustalW and ClustalX. Curr Protoc Bioinformatics. 2002;Chap. 2, Unit 2.3.

44. Tamura K, Stecher G, Peterson D, Filipski A, Kumar S. MEGA6: molecular evolutionary genetics analysis version 6.0. Mol Biol Evol. 2013;30(12):2725-2729.

45. Trapnell C RA, Goff L, Pertea G, Kim D, Kelley DR. Differential gene and transcript expression analysis of RNA-seq experiments with TopHat and Cufflinks. Nat Protoc. 2012;7(3):562-578.

46. Trapnell C, Hendrickson DG, Sauvageau M, Goff L, Rinn JL, Pachter L. Differential analysis of gene regulation at transcript resolution with RNAsEq. Nat Biotechnol. 2013;31(1):46-53.

47. Yoo SD, Cho YH, Sheen J. Arabidopsis mesophyll protoplasts: a versatile cell system for transient gene expression analysis. Nat Protoc. 2007;2(7):1565-1572.

48. Clough SJ, Bent AF. Floral dip: a simplified method for Agrobacterium-mediated transformation of Arabidopsis thaliana. Plant J. 1998;16(6):735-743.

\section{Figures}

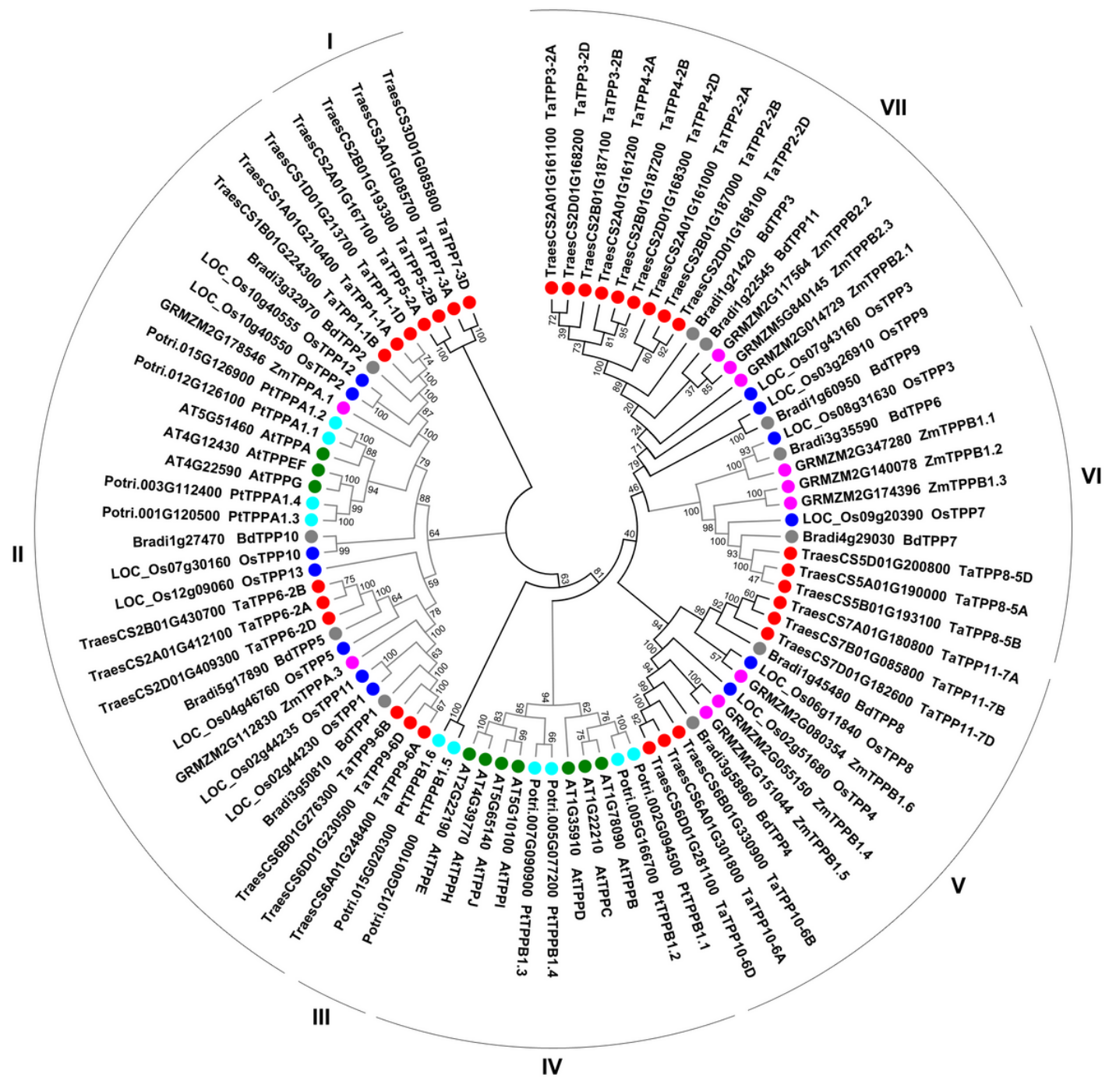

Figure 1

Phylogenetic relationships of the TPP family in plants The phylogenetic tree of TPP proteins from wheat, maize, rice, Populus, Arabidopsis, and B. distachyum. AMEGA 6.0 was used to build a neighbor-joining tree, which was visualized with the online tool Evolview. TPPs from one plant species 
are marked with leaf labels. I-VII denotes the seven plant TPP protein subfamilies. The percentage bootstrap scores were calculated from 1000 replications.

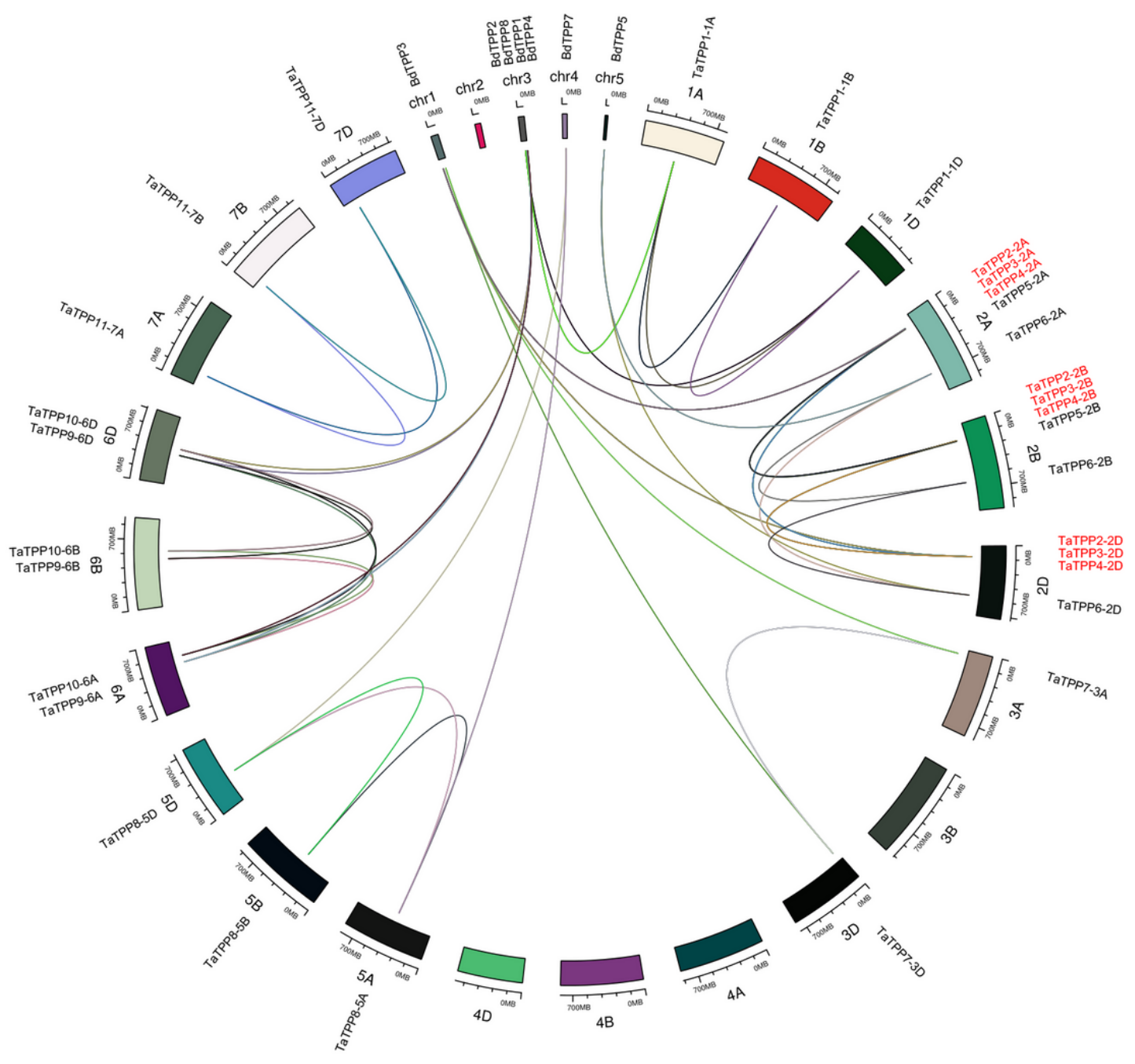

Figure 2

Mapping and analysis of synteny of TaTPP genes B. distachyum and wheat chromosomes are represented as circles. The location of each BdTPP and TaTPP gene is denoted with a small black line on the circle. Colored curves indicate syntenic relationships between B. distachyum and wheat TPPs genes. 
A

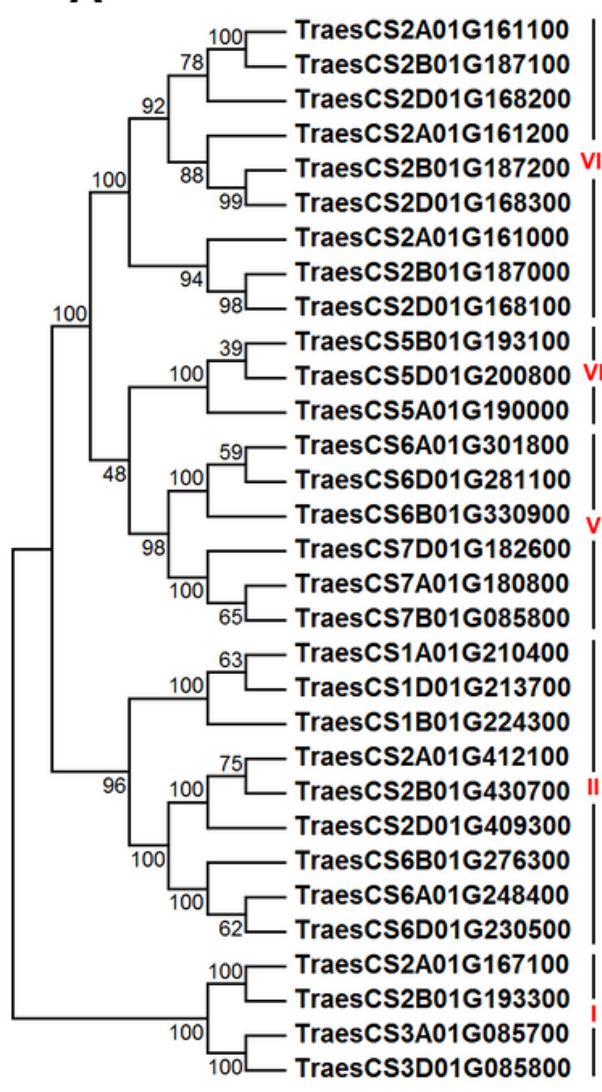

B

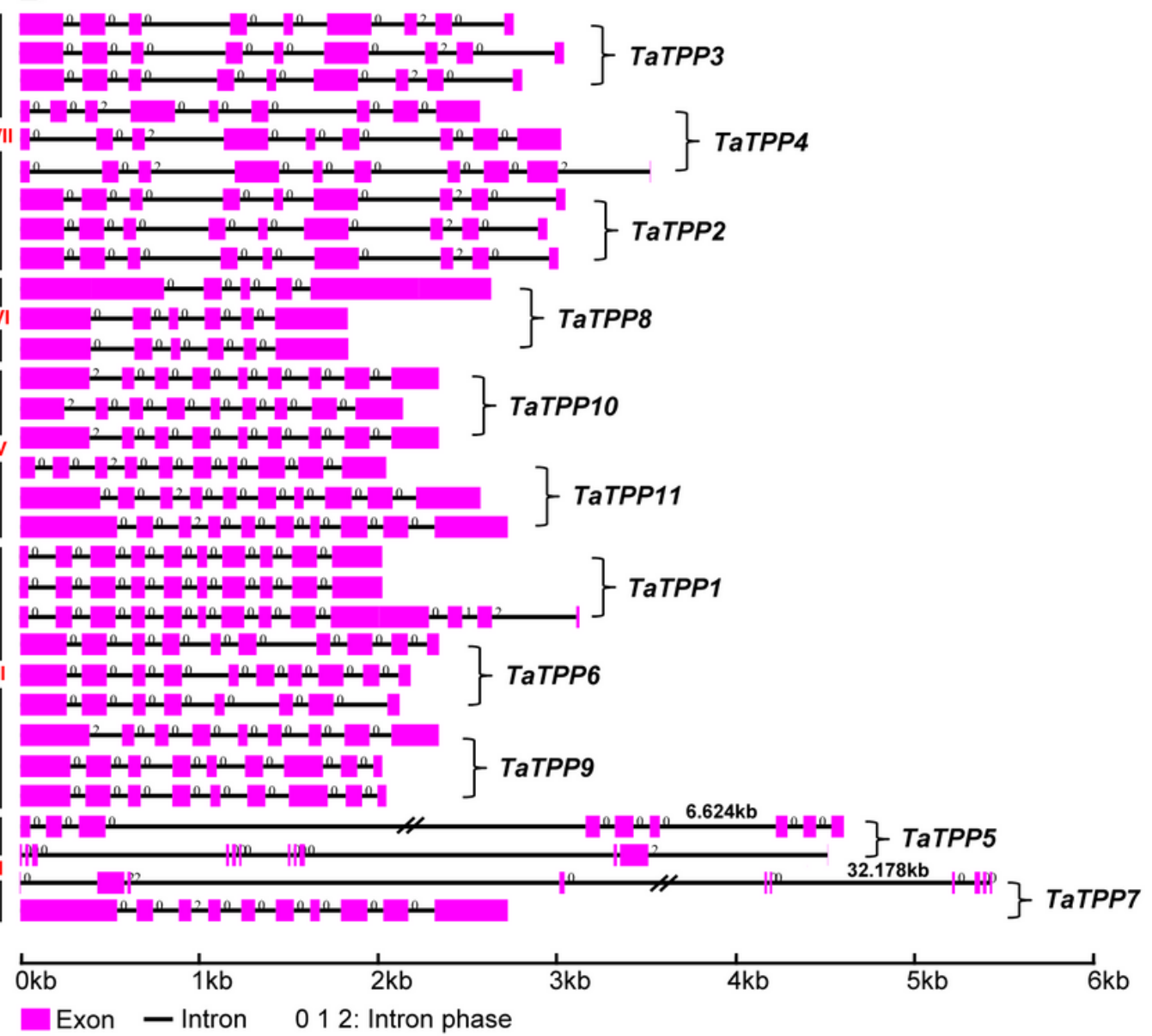

Figure 3

Gene structures and phylogenetic relationships of wheat TPP genes (A) Phylogenetic tree of 31 full-length wheat TPP proteins generated with MEGA 6.0 and the Neighbor-Joining (NJ) method with 1,000 bootstrap values. (B) Exon/intron structures of TPP genes in wheat. Exons and introns denoted by purple boxes and black lines, respectively. 


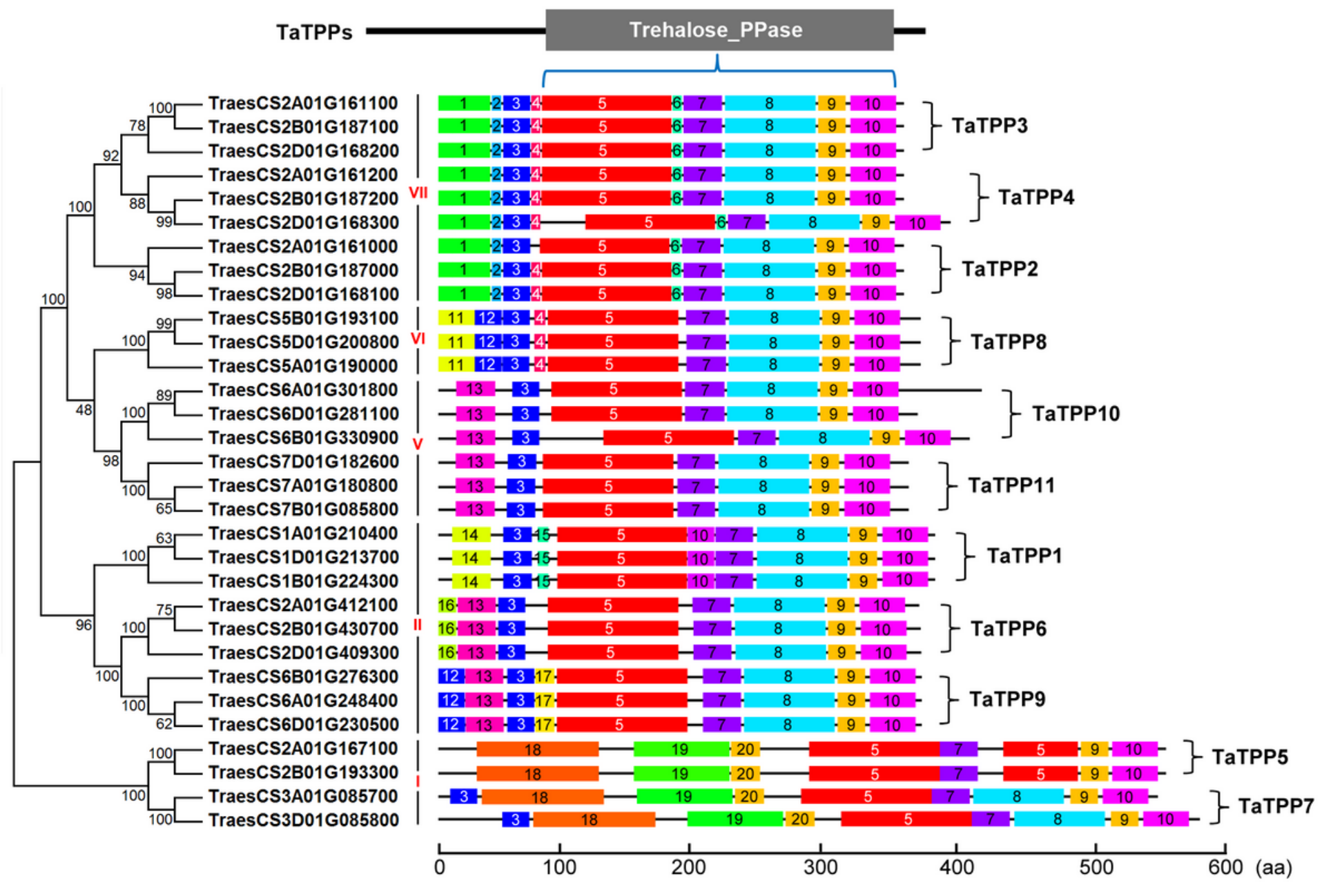

Figure 4

Conserved motif analysis of TPP proteins in wheat A colored box denotes each motif, while black lines indicate non-conserve sequences. Conserved TPP domains consist of motifs 4-10. 


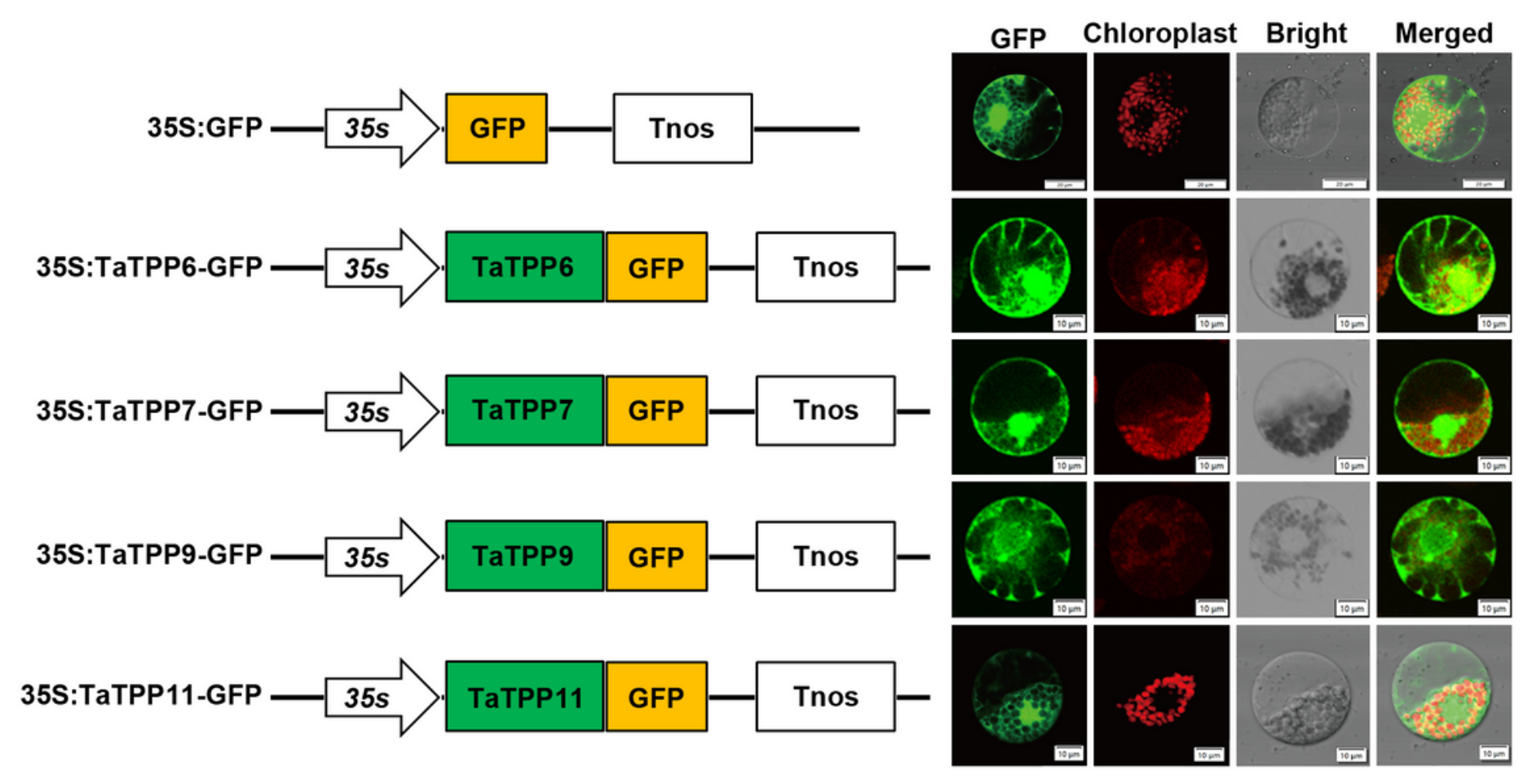

Figure 5

Subcellular localization of TaTPP-GFP fusion proteins in wheat mesophyll protoplasts The 35S::TaTPP6-GFP, 35S::TaTPP7-GFP, 35S::TaTPP9-GFP, and 35S::TaTPP11-GFP fusion vectors, and 35S::GFP control vectors were all independently transformed into wheat mesophyll protoplasts via PEG transfection. A laser scanning confocal microscope was used to observe the green fluorescence. 


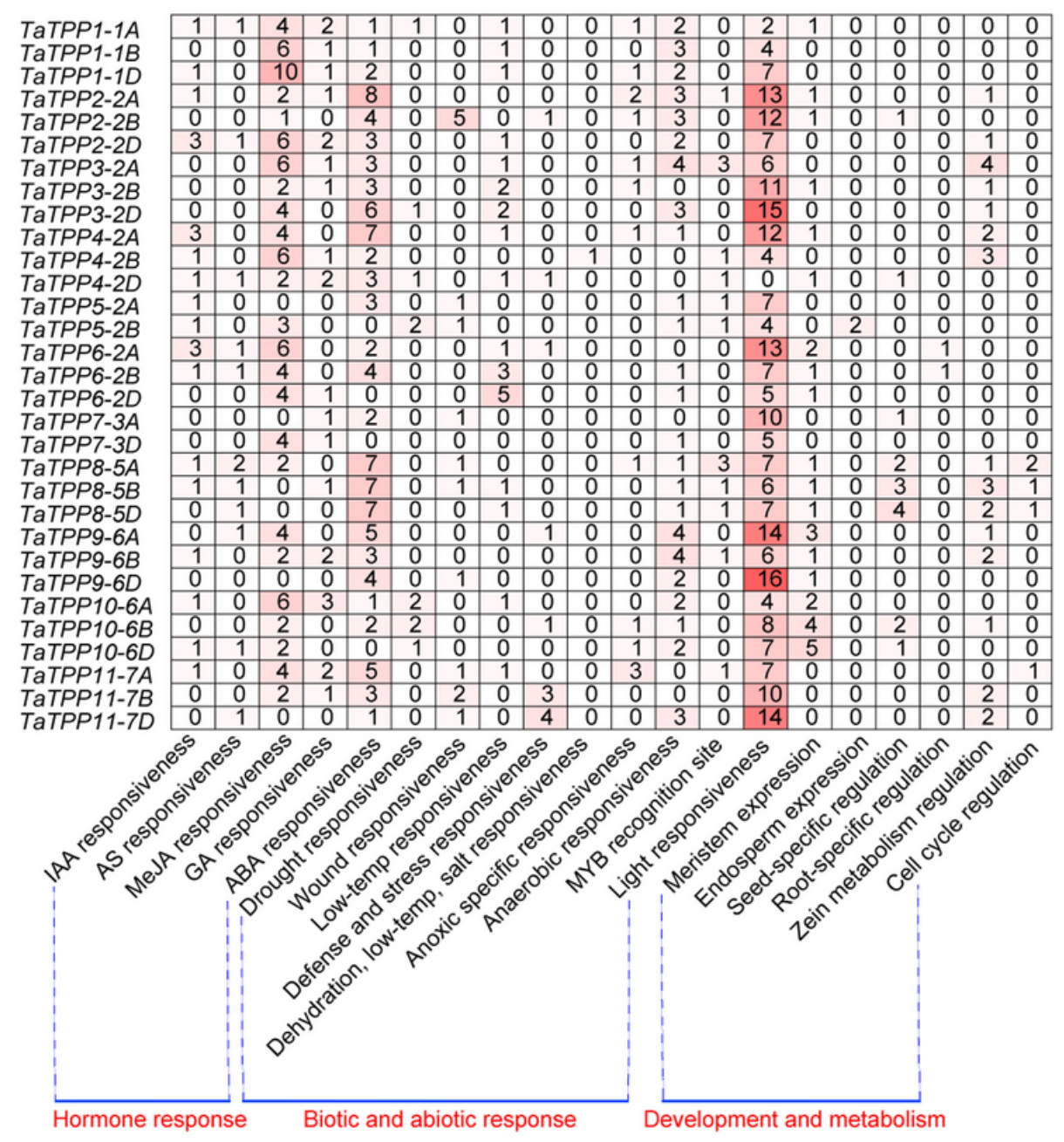

\section{Figure 6}

Promoter cis-element analysis of TaTPP genes 2-kb upstream promoter sequence for all TaTPP genes was obtained from wheat genome database, while PlantCARE was used to scan all the cis-acting regulatory elements. Numbers denote the sum of how different cis-acting elements respond to similar stimuli. 


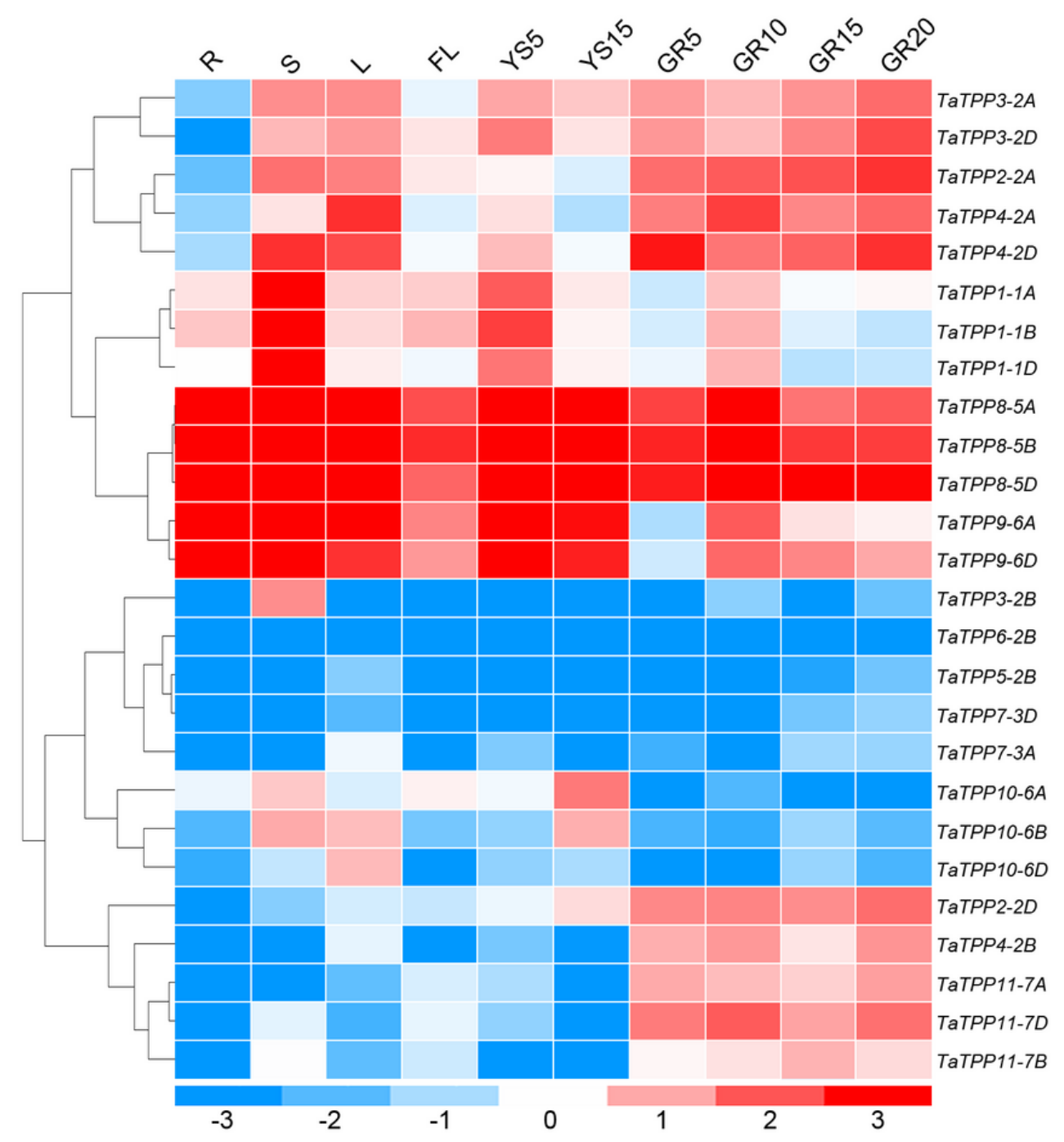

Figure 7

TaTPP gene expression profiles in ten different organs or tissues Heatmap drawn from Log10-transformed expression values. Blue or red indicates the lower or higher levels of expression of each transcript in each sample, respectively. $R$, root of wheat seedling at five-leaf stage; S, stem of wheat seedling at five-leaf stage; L, leaf of wheat seedling at five-leaf stage; FL, flag leaf at heading stage; YS5, young spike at early booting stage; YS15, spike at heading stage; GR5, grain of five days post-anthesis; GR10, grain of 10 days post-anthesis; GR15, grain of 15 days post-anthesis; GR20, grain of 20 days post-anthesis. 

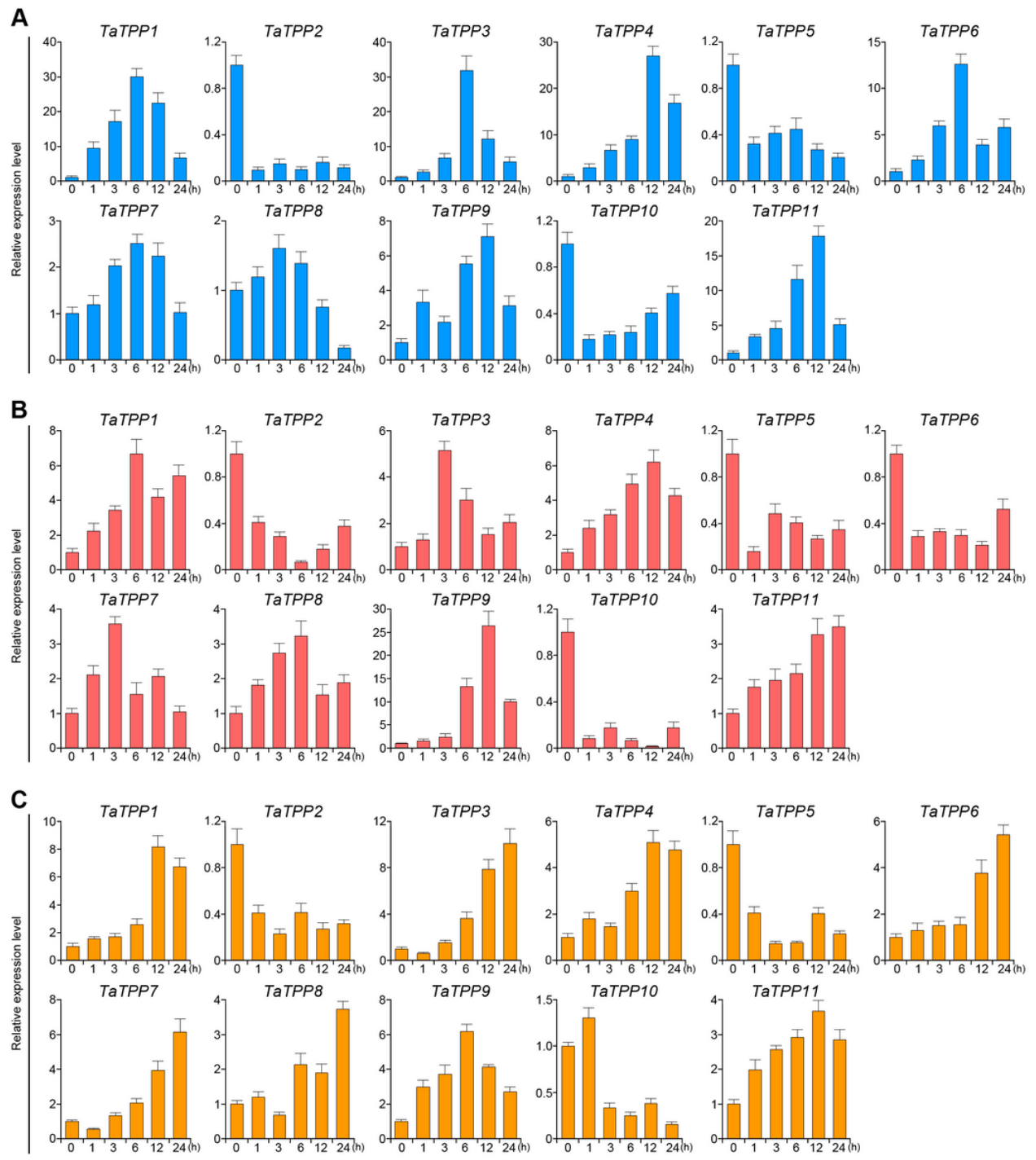

Figure 8

TaTPP gene expression profiles responding to abiotic stresses (A) TaTPP gene expression patterns after ABA treatment. (B) TaTPP gene expression pattern under low-temperature treatment. (C) TaTPP gene expression pattern under salt stress treatment. 

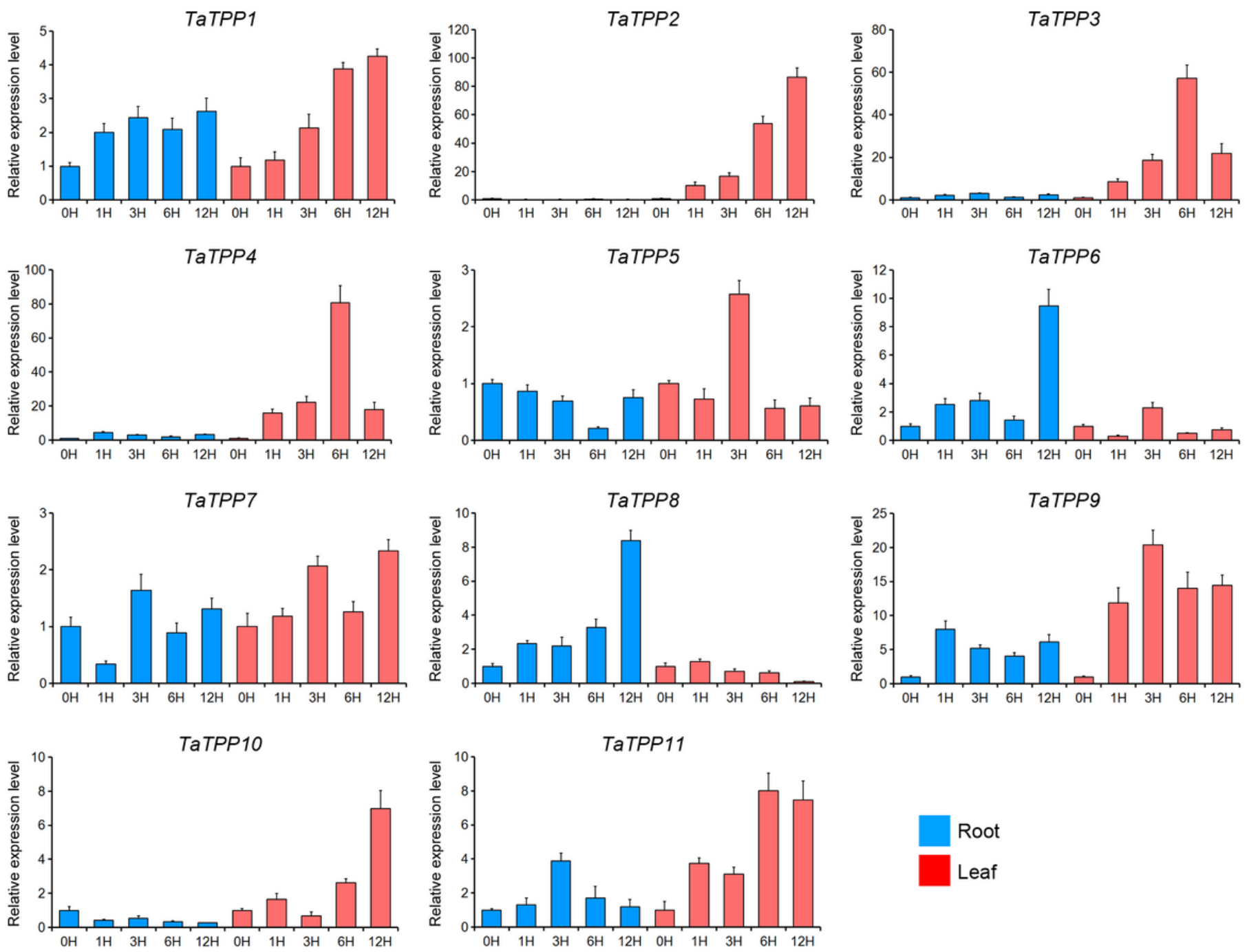

Figure 9

TaTPP gene expression profile in seedling roots and leaves under drought stress conditions Red and blue columns represent level of TaTPP gene expression under $20 \%$ PEG treatment in plant leaves and roots obtained from wheat seedlings, respectively. X-axis indicates time points following drought treatment. Expression data from the control sample were normalized to 1, while error bars indicate standard error from three replicates. 

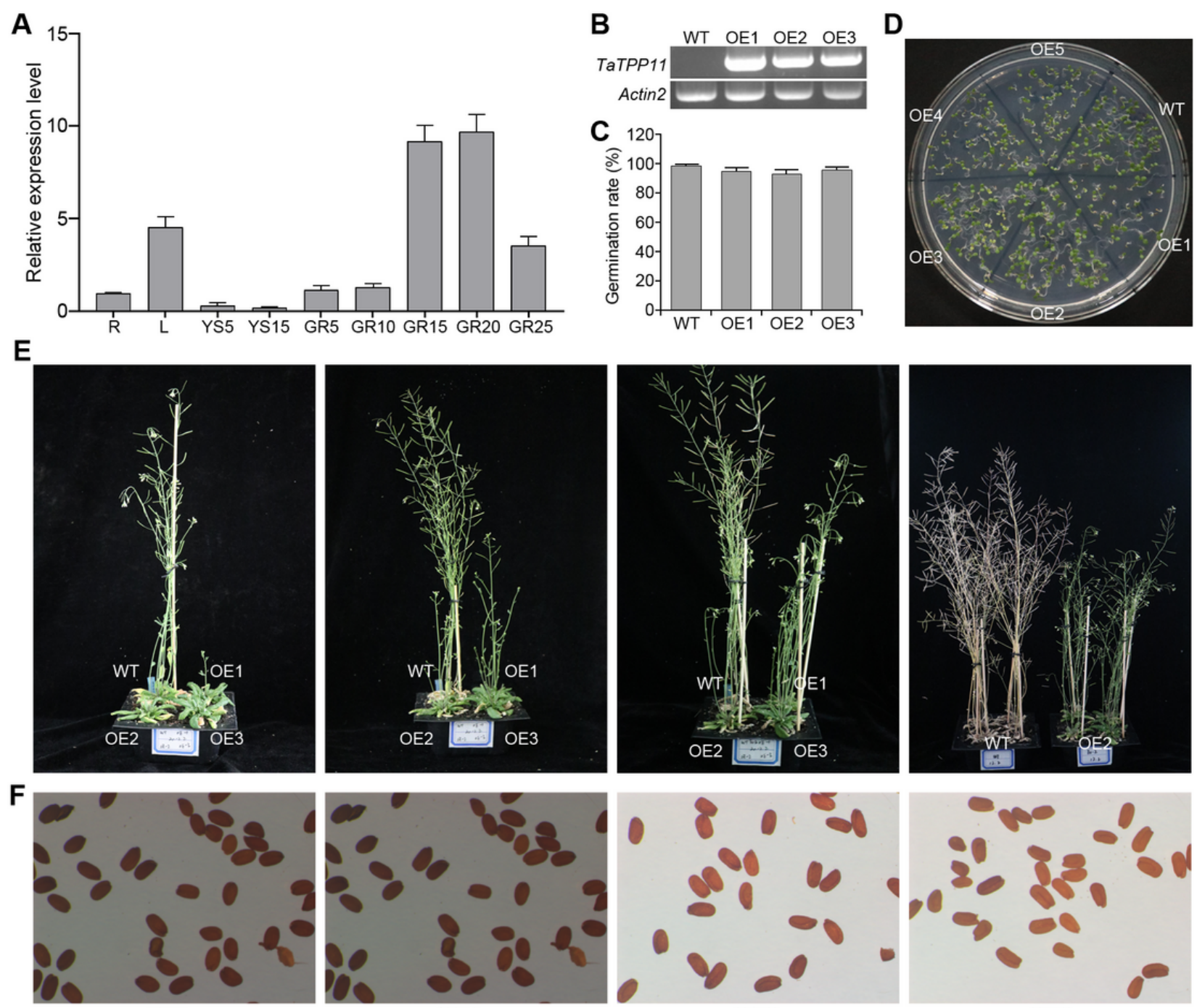

WT

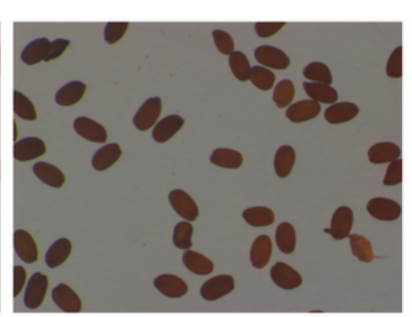

OE1
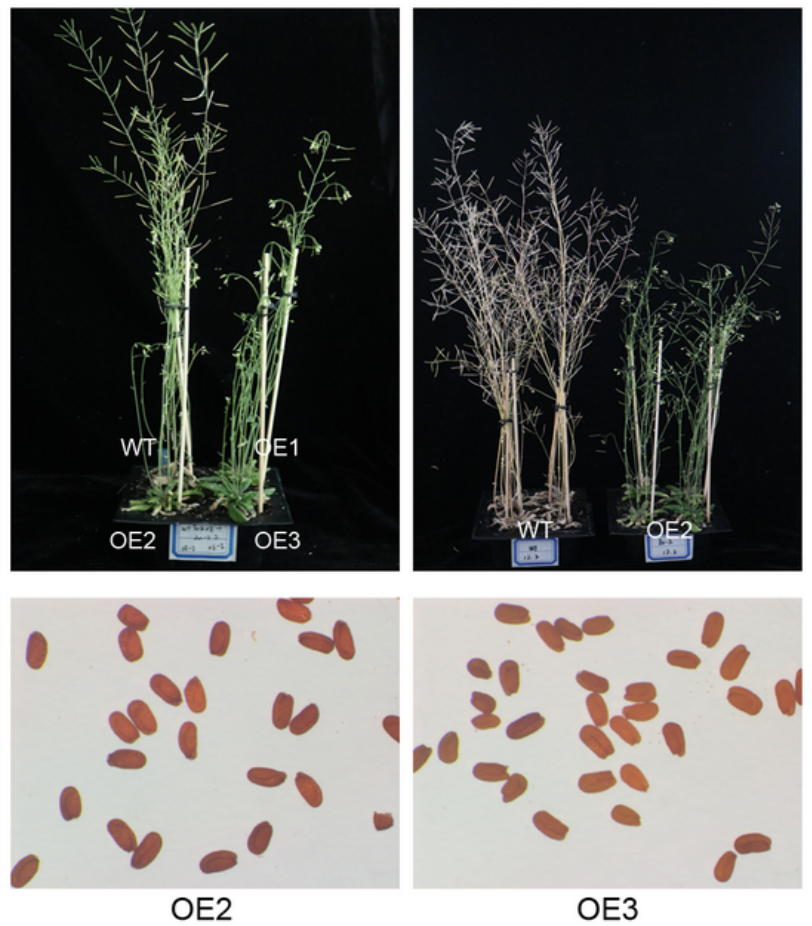

Figure 10

Comparing plant morphology between WT plants and TaTPP11 overexpression (A) TaTPP11 expression profiles in various tissues. R, root of wheat seedling at five-leaf stage; L, leaf of wheat seedling at five-leaf stage; YS5, young spikes of 5 days after booting; YS15, spikes 15 days after booting; GR5, grain of five days post-anthesis; GR10, grain of 10 days post-anthesis; GR15, grain of 15 days post-anthesis; GR20, grain of 20 days postanthesis; GR25, grain of 25 days post-anthesis. (B) RT-PCR identification of Arabidopsis lines overexpressing TaTPP11-7D. (C-D) Seed germination assay between 35S:TaTPP11-7D transgenic and WT plants. (E-F) Plant phenotype (E) and seed morphology (F) of wild-type and 35S:TaTPP11-7D transgenic Arabidopsis plants. 
A
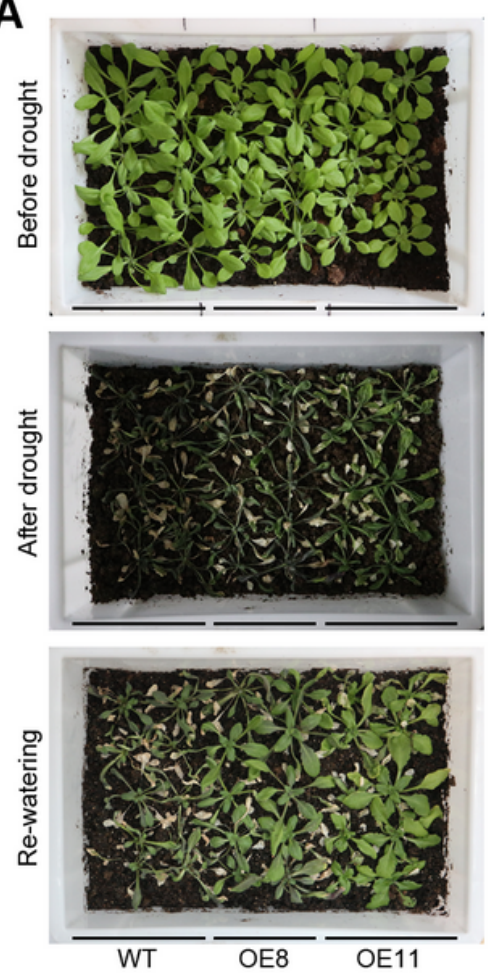

B
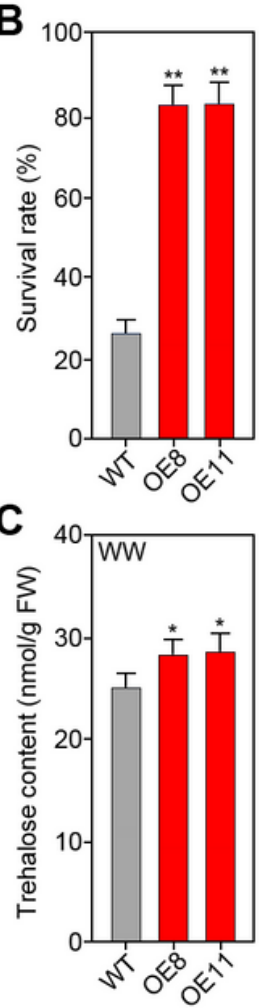
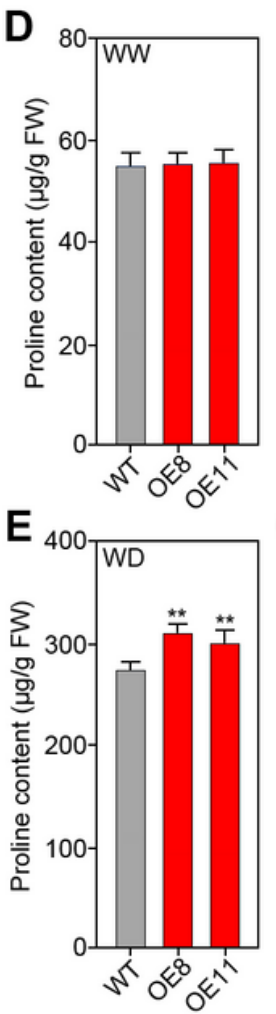
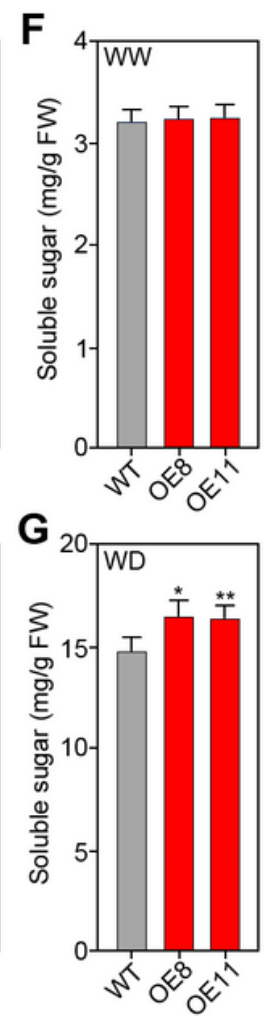
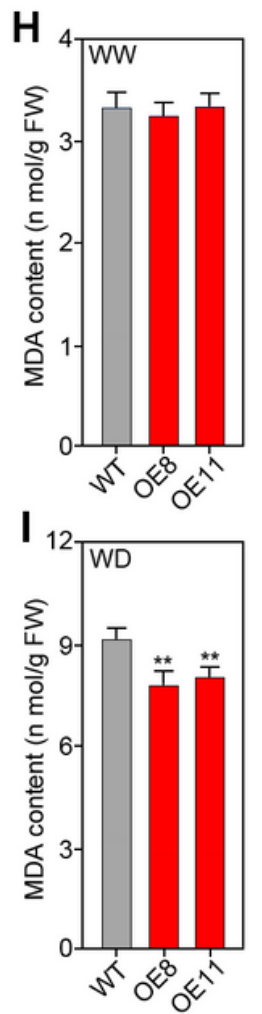

\section{Figure 11}

Drought stress tolerance of Arabidopsis plants overexpressing TaTPP11. (A) Phenotypes of 35S:TaTPP11-7D transgenic Arabidopsis and wild-type (WT) plants before and after drought stress. (B) Statistical analysis of survival rates following drought-stress treatment. Survival rates and standard errors were obtained using data acquired from four independent experiments. Each experiment used 12 plants for each line. (C) Trehalose contents in WT and 35S:TaTPP11-7D transgenic lines. (D-I) Proline, soluble sugar, and MDA contents in WT and 35S:TaTPP11-7D transgenic lines under well-watered (WW) and water-deficit (WD) conditions. Values indicates mean \pm SD of three replicates. Asterisks represent significant differences from the WT per on student's t-test $\left({ }^{*} P<0.05\right.$; $\left.{ }^{*} P<0.01\right)$.

\section{Supplementary Files}

This is a list of supplementary files associated with this preprint. Click to download.

- Additionalfile1Tables1.xlsx

- Additionalfile2Figures1.tif

- Additionalfile3Figures2.tif

- Additionalfile4Figures3.tif

- Additionalfile5Tables2.xlsx

- Additionalfile6Figures4.tif

- Additionalfile7FigureS5.tif

- Additionalfile8TableS3.xlsx 